\title{
STATISTICAL ANALYSIS OF THE MARIANA TRENCH GEOMORPHOLOGY USING R PROGRAMMING LANGUAGE
}

\author{
Polina LEMENKOVA $\mathbb{C}^{*}$ \\ College of Marine Geoscience, Ocean University of China, Qingdao, China
}

Received 10 July 2018; accepted 03 June 2019

\begin{abstract}
This paper introduces an application of $\mathrm{R}$ programming language for geostatistical data processing with a case study of the Mariana Trench, Pacific Ocean. The formation of the Mariana Trench, the deepest among all hadal oceanic depth trenches, is caused by complex and diverse geomorphic factors affecting its development. Mariana Trench crosses four tectonic plates: Mariana, Caroline, Pacific and Philippine. The impact of the geographic location and geological factors on its geomorphology has been studied by methods of statistical analysis and data visualization using $\mathrm{R}$ libraries. The methodology includes following steps. Firstly, vector thematic data were processed in QGIS: tectonics, bathymetry, geomorphology and geology. Secondly, 25 cross-section profiles were drawn across the trench. The length of each profile is $1000-\mathrm{km}$. The attribute information has been derived from each profile and stored in a table containing coordinates, depths and thematic information. Finally, this table was processed by methods of the statistical analysis on R. The programming codes and graphical results are presented. The results include geospatial comparative analysis and estimated effects of the data distribution by tectonic plates: slope angle, igneous volcanic areas and depths. The innovativeness of this paper consists in a cross-disciplinary approach combining GIS, statistical analysis and R programming.
\end{abstract}

Keywords: R, statistical analysis, programming, Mariana Trench, oceanography, geomorphology.

\section{Introduction}

The goal of this study is to analyze factors affecting geomorphological structure of the Mariana Trench. Mariana Trench is a long and narrow topographic depression of the seafloor located in the west Pacific ocean, $200 \mathrm{~km}$ to the east of the Mariana Islands, east of the Philippines.
It is known as the deepest part of the ocean floor reaching a maximum-known depth of $10.984 \pm 25 \mathrm{~m}(95 \%)$ at $11.329903^{\circ} \mathrm{N} / 142.199305^{\circ} \mathrm{E}$ (Figure 1) at the Challenger Deep (Gardner, Armstrong, Calder, \& Beaudoin, 2014). It has a distinctive morphological feature of the convergent Pacific plate boundaries, along which lithospheric plates

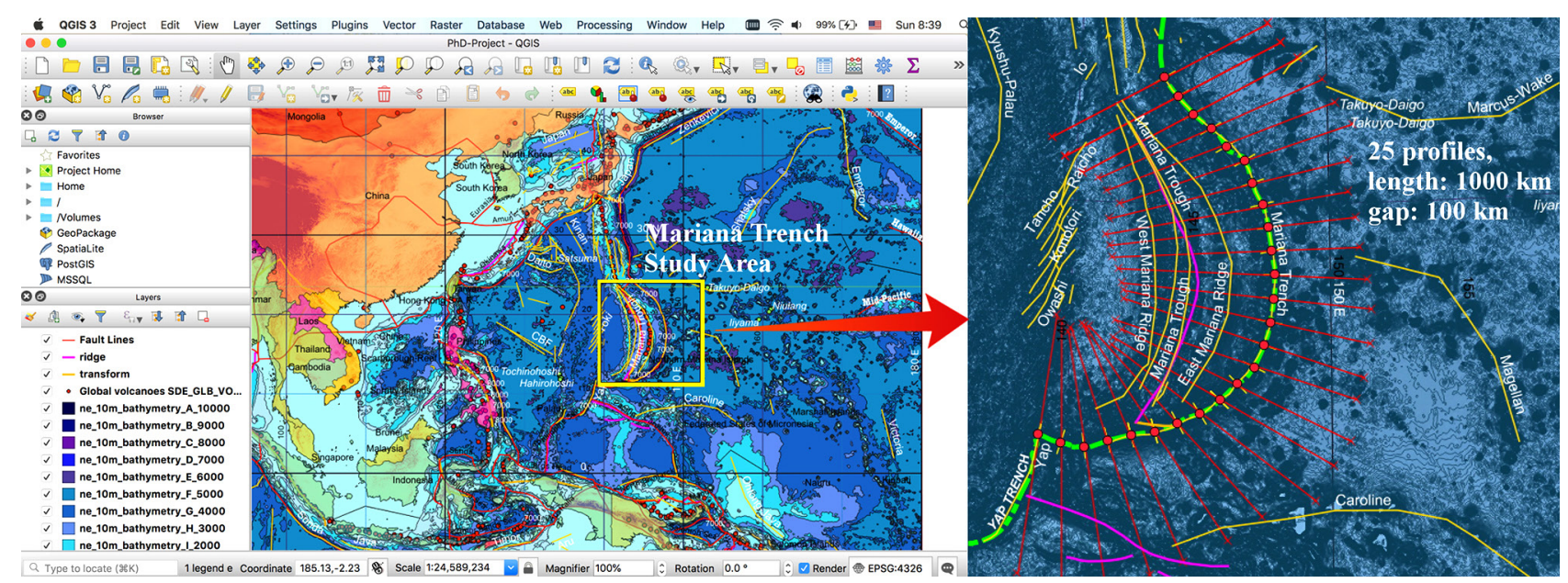

Figure 1. Study area: Mariana Trench. Visualization: QGIS 3.0

*Corresponding author. E-mail: pauline.lemenkova@gmail.com

This is an Open Access article distributed under the terms of the Creative Commons Attribution License (http://creativecommons.org/licenses/by/4.0/), which permits unrestricted use, distribution, and reproduction in any medium, provided the original author and source are credited. 
move towards each other. The total length of the trench measures ca. $2.550 \mathrm{~km}$ long on average.

\section{General characteristics of the Mariana Trench}

\subsection{Processes of the deep-sea sedimentation}

Processes of the deep-sea terrigenous sedimentation are formed by the transfer of the erosion materials from the adjacent land. The main processes include transportation, deposition and re-deposition of the sedimentation materials. The origin of the deep ocean sedimentation is diverse. In general, apart from the sediments from the rivers, the terrigenous material goes to the oceans through iceberg melting and glacial deposits transferred to the seafloor, as well as dusty material moved by the aeolian processes. Normally, the sediments transferred by rivers are deposited within the shelf sublittoral areas, and rarely reach deeper regions of the continental slope and, even less the abyssal basins. However, sediments deposited on the shelf may move to the deeper parts of the ocean including hadal trenches, due to further move from the shelf edge downwards, avalanche sedimentation, i.e. gravitational flows, which are caused by the gravity. For the case of the Mariana Trench, the deep currents flow through the trench ventilating its bottom water flowing through the West Pacific trenches originates from the Southern Ocean and flows northward (Warren \& Owens, 1988), in a clockwise direction, passing through the trenches on the west of the Pacific (i.e. the Kermadec Trench and the Tonga Trench. Through the Samoan Passage, it flows northwest across the equator to the east Mariana Trench. Among other hadal trenches, Mariana Trench is the largest structural traps located in the continental margins. It stretches for hundreds kilometers and has a narrow depression on the ocean floor with very steep slopes and the depths of $3-5 \mathrm{~km}$ width of the bottoms rarely exceeding $5 \mathrm{~km}$ in breadth (Nakanishi \& Hashimoto, 2011).

Therefore, the geomorphic conditions of Mariana Trench create ideal conditions for the sedimentation processes intercepting almost all clastic material originating from adjacent land. The accumulation of the terrigenous sedimentation on the Mariana Trench seafloor depends on various factors. These include climatic and hydrodynamic local characteristics, tectonics, structure of the seafloor bathymetry, geomorphic characteristics of the trench, type and size of particles and others.

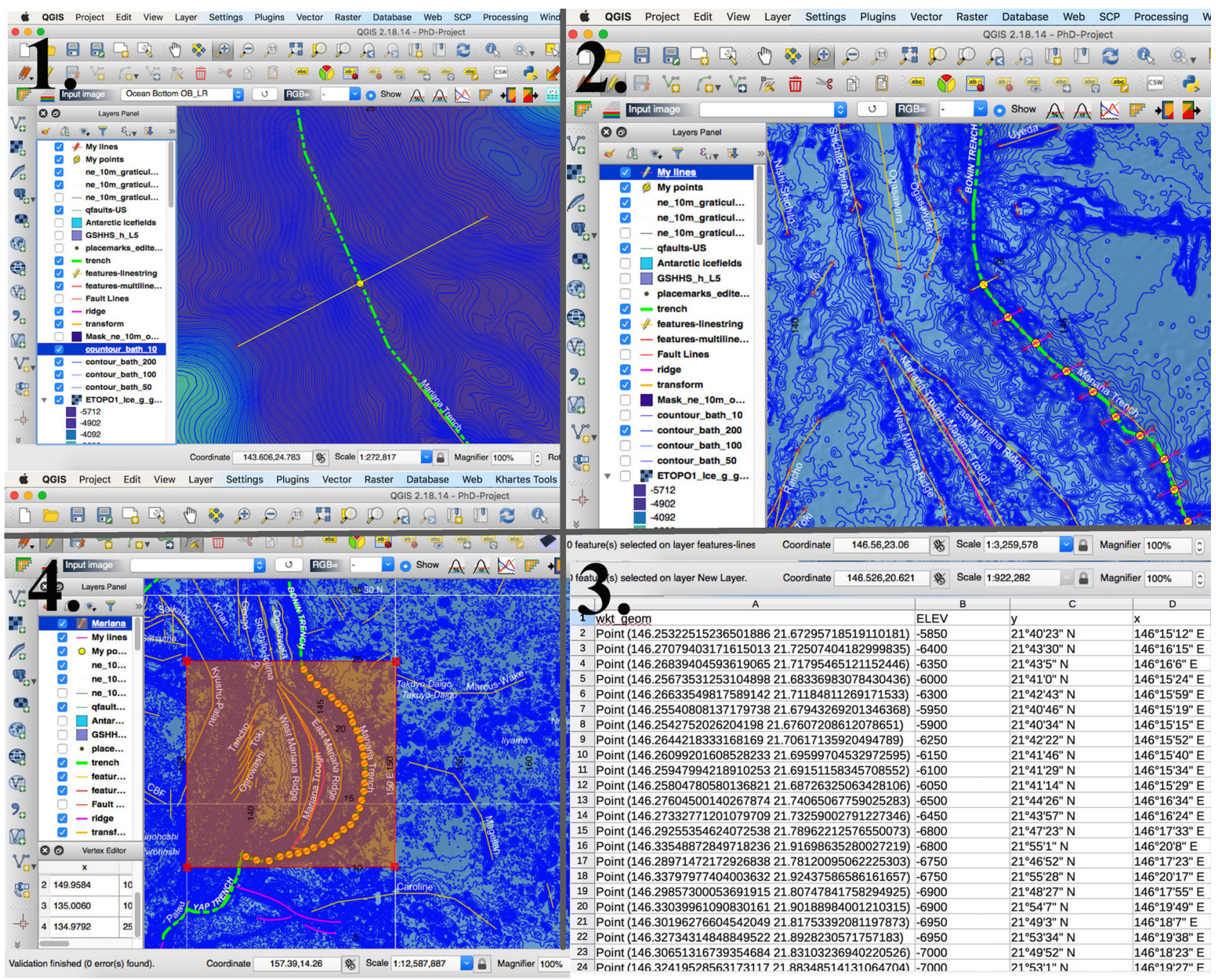

Figure 2. Stepwise illustration of the creating a series of 25 bathymetric profiles, Mariana Trench, Quantum GIS 3.0 

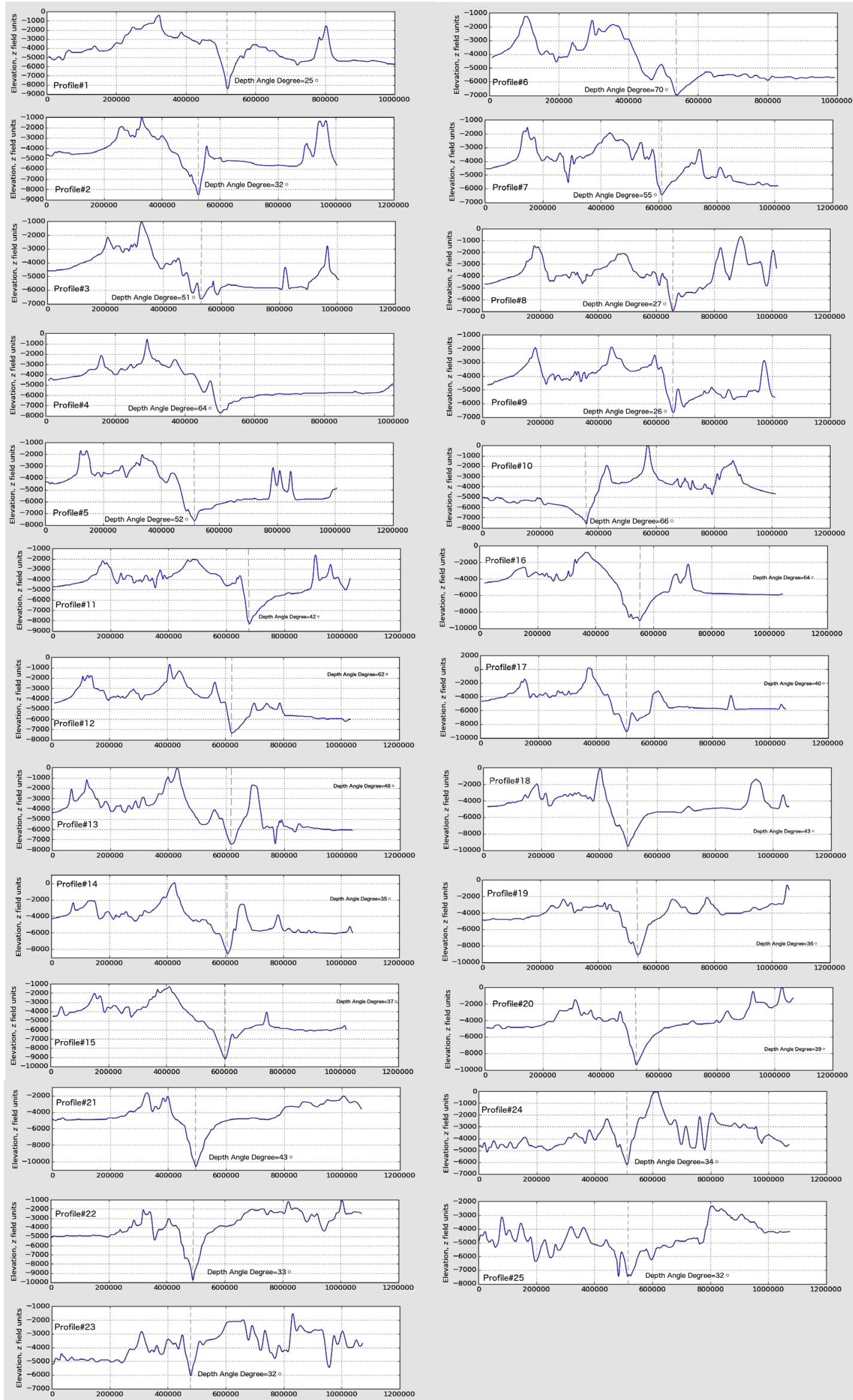

Figure 3. Graphs of the 25 bathymetric profiles, Mariana Trench, Quantum GIS 3.0 


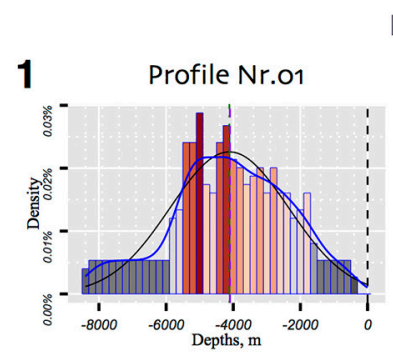

Mariana Trench Bathymetry: Histograms of Depth Distribution
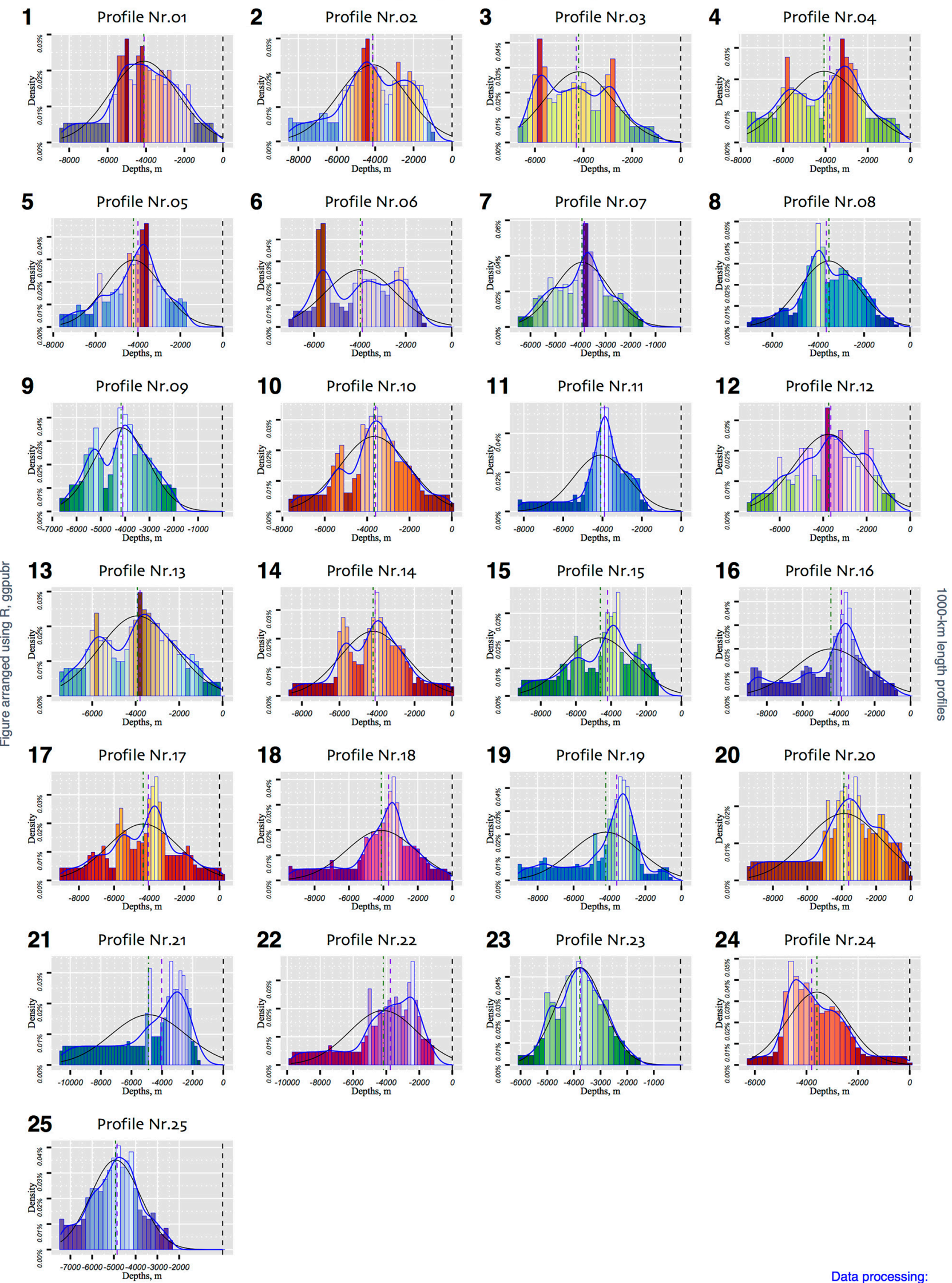
Mariana Trench, Profiles Nr.1-25

Local Polynomial Regression, Confidence Interval and Quantiles QQ

(LOESS method: locally weighted scatterplot smoothing for non-parametric regression)
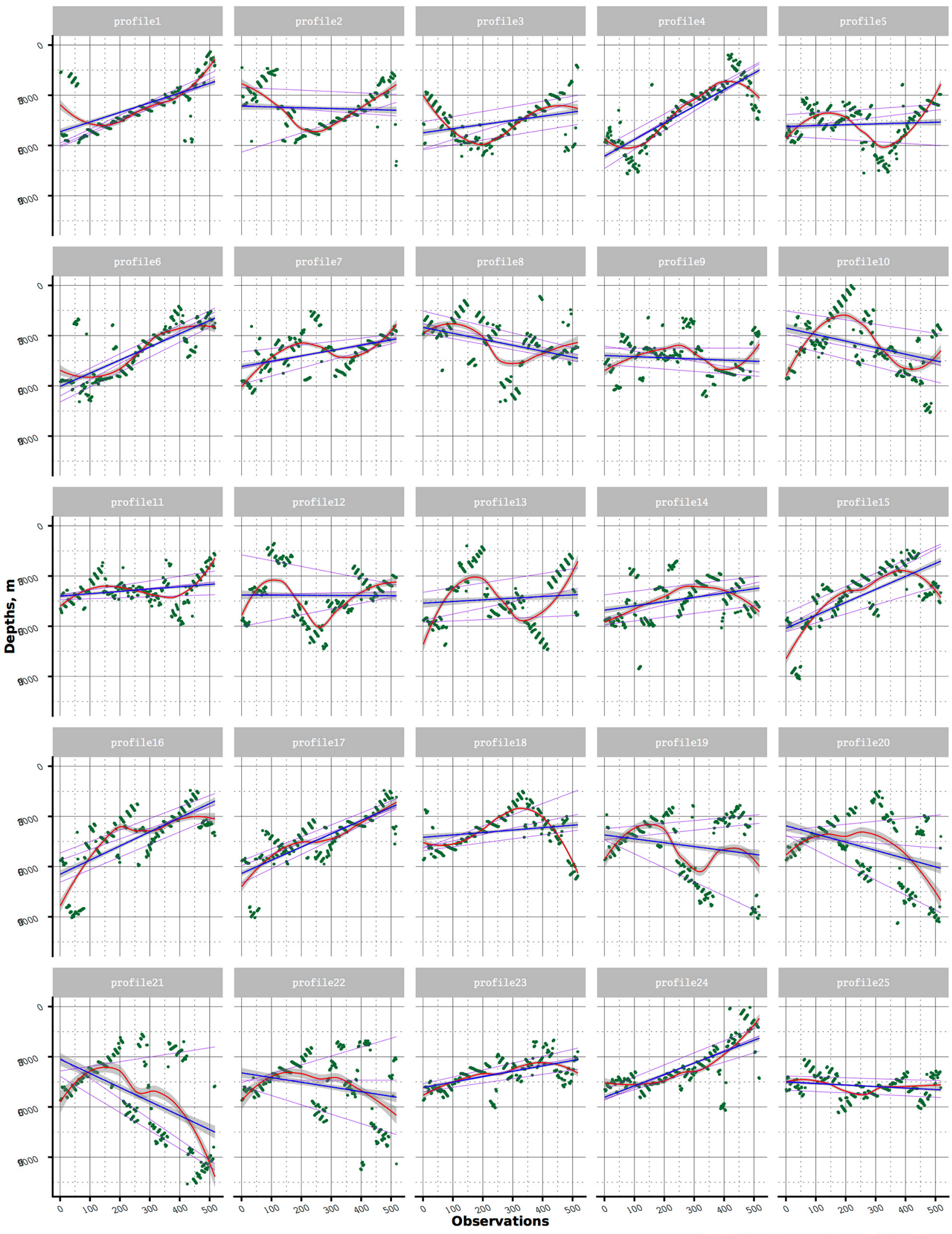

Statistics Processing and Graphs: R Programming. Data Source: QGIS 
Large areas of active sedimentation processes can be distinguished in the areas where the speed of the sedimentation and their volumes are very high, as well as local areas of active sedimentation, or, on the contrary, dominating erosion areas. In general, sedimentation processes mainly occur in various sediment traps, however, some areas are noted by areal sedimentation. Therefore, the processes of the sedimentation within the Mariana Trench are highly uneven.

\subsection{Bathymetry and the system of crack}

Mariana Trench presents a strongly elongated, arched in plan and lesser rectilinear depression. Its transverse profile is asymmetric: the slope is higher on the side of the Mariana island arc. The slopes of the trench are dissected by deep underwater canyons. Various narrow steps are also often found on the slopes of the trench. On the longitudinal profiles of the bottom of the Mariana there are many deepwater detected transverse steps and thresholds connected by the movements of the earth's crust along transverse faults (Zhou, Lin, \& Behn, 2015).

These thresholds can play important role in the evolution of the deep water trough, for example, to control the intensity of its filling by sedimental precipitation. In this process very important are the streams of the precipitation moving along the bottom from the part of the trough
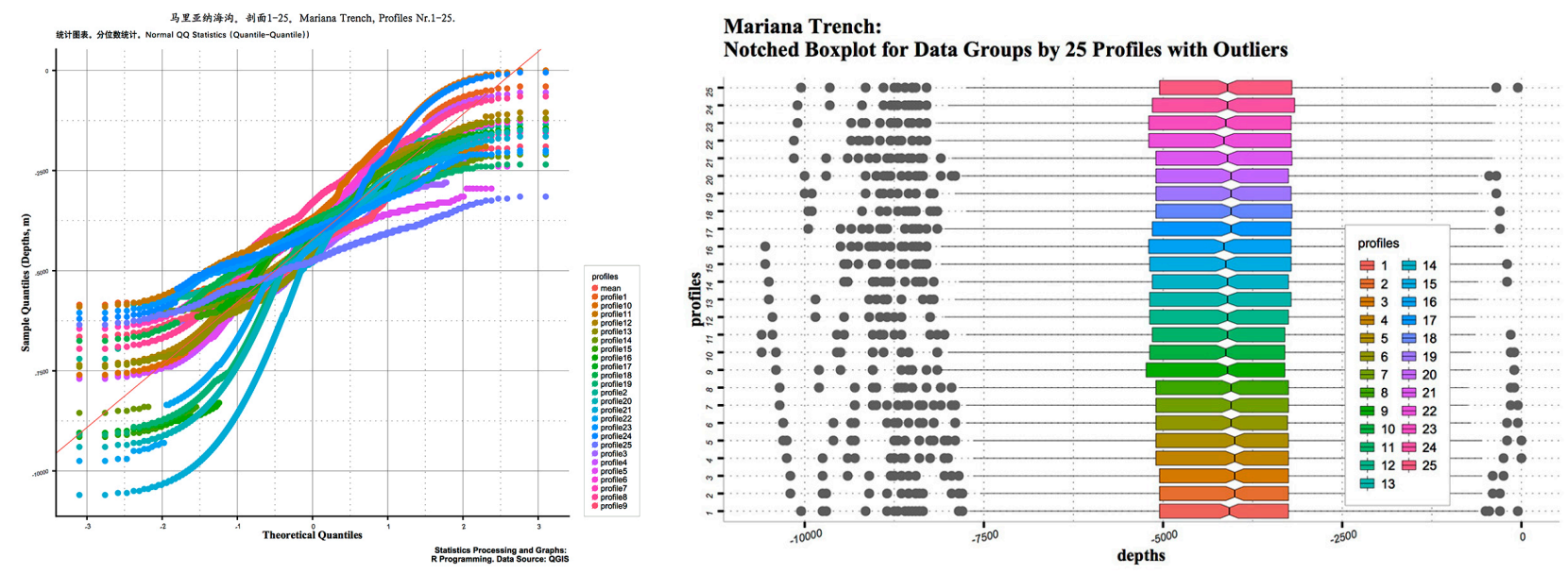

Figure 6 Quantile statistics (QQ), empirical cumulative density function (ECDF) and notched plot

A

Mariana Trench

Strip Plot: Slope Angle(tg(A/H)) in 25 Profiles by Tectonic Plates

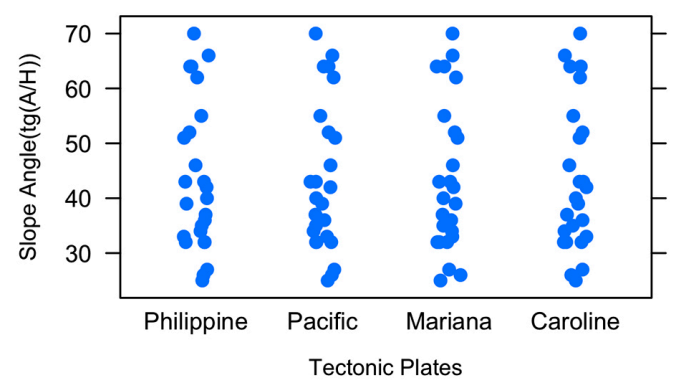

C

Mariana Trench

Strip Plot: Maximal Depth in 25 Profiles by Tectonic Plates

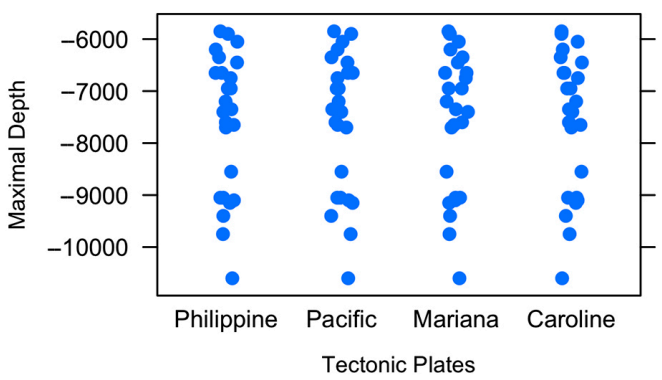

B

Strip Plot: Sedimental Thickness in 25 Profiles by Tectonic Plates

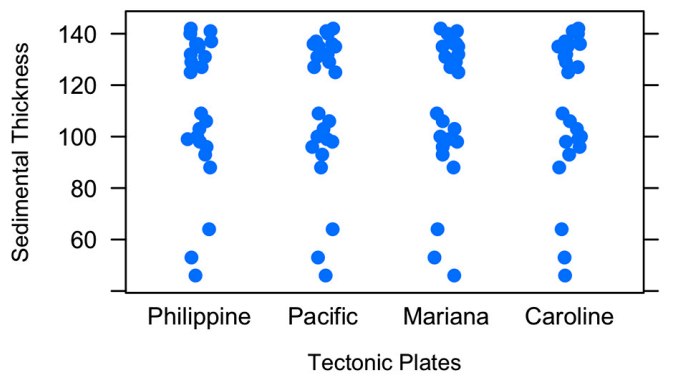

D

\section{Mariana Trench}

Strip Plot: Igneous Volcanic Zones Distribution in 25 Profiles by Tectonic Plates

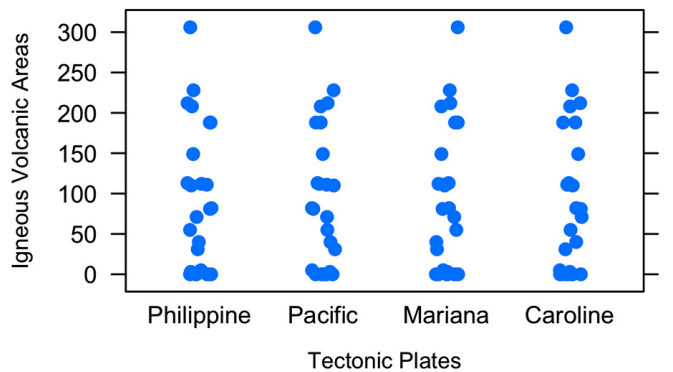

Figure 7. Distribution of slope angle, sedimental thickness, depth and igneous volcanic zones, by four tectonic plates 


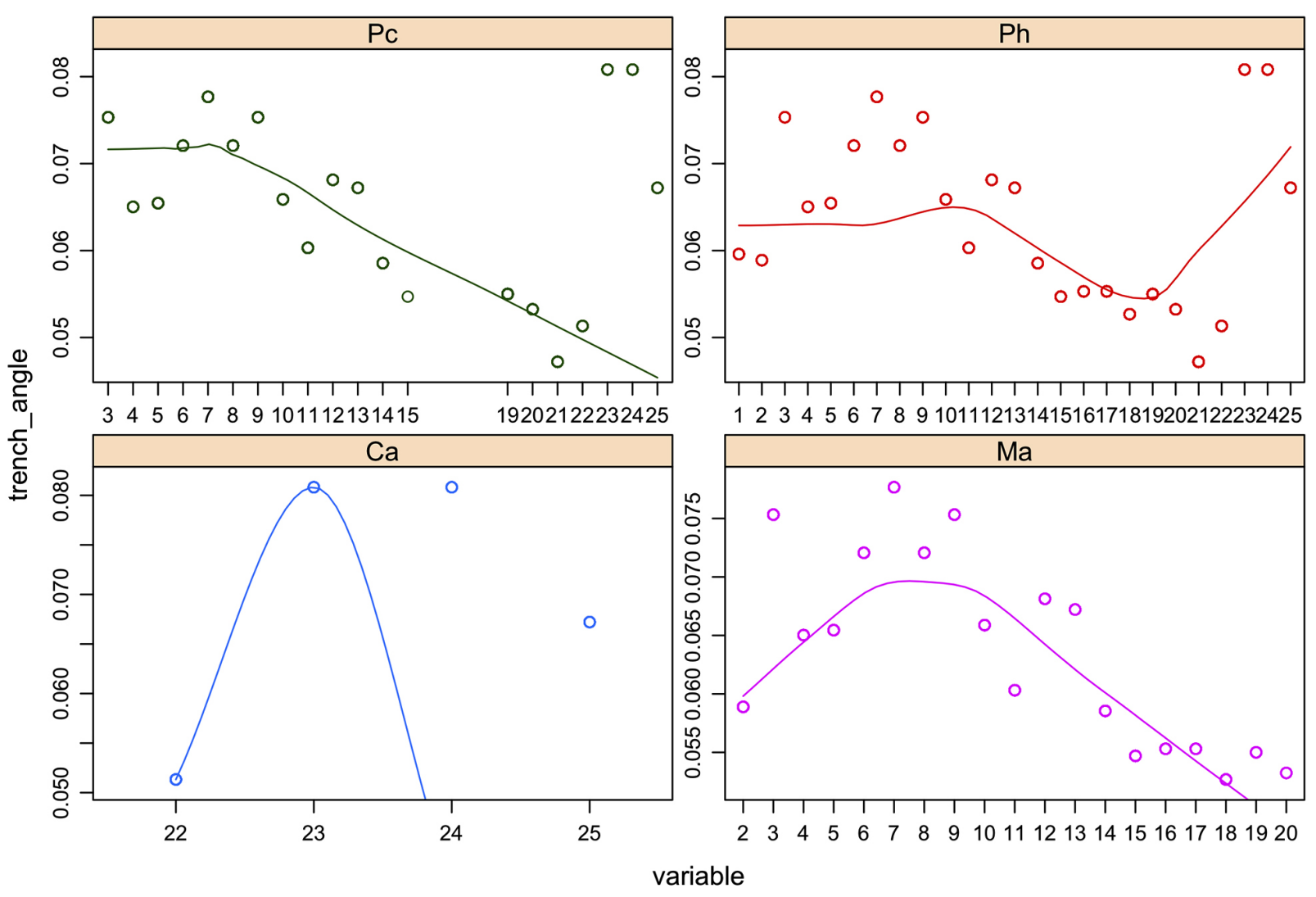

Figure 8. Multiple panel by groups: four tectonic plates, trench slope angle in the deepest point

which is closer to the continent (Taira et al., 2004). At the bottom of the trough there is a transverse threshold, which can present kind of a dam, in front of which there is accumulation of a thick layer of sediment precipitation.

Mariana Trench has complicated steps of various shapes and sizes, caused by active tectonic and sedimental processes. The steepness of its geomorphic depth averages in 4-5 degrees, but its individual parts can be limited to steeper slopes as subjects to the gravitational flow system of the submarine canyons and valleys (Kawabe, 1993). Complex distribution of the various geomorphic material on the adjacent abyssal plains of the ocean contributes to the formation of the geomorphic features of the particular region of the Mariana Trench.

\subsection{Igneous volcanic areas}

Mariana Trench is an important integral feature of the active continental margins that naturally includes deep plate subduction. Subduction of slabs of oceanic lithosphere into the deep mantle involves a wide range of the geophysical and geochemical processes and is of major importance for the physical and chemical evolution of the Earth (Miller, Kennett, \& Lister, 2004). Thus, the subduction and subduction-related volcanism are the major processes through which geochemical components are being recycled between the Earth's crust, lithosphere and mantle (Yoshioka, Torii, \& Riedel, 2015). A large proportion of the world's earthquakes and volcanoes are related to the subduction (Kirby, Stein, Okal, \& Rubie, 1996). Deep sea volcanism results from a range of processes including dehydration, melting and melt migration. The deepest known earthquakes occur in the subducted lithosphere at depths of $660-700 \mathrm{~km}$ but their cause is still not explained. In addition, the motion and velocities of the lithospheric plates at the Earth's surface are controlled largely by the buoyancy forces that drive subduction. Because of the wide variety of processes involved, subduction zones can be regarded as natural laboratories through which dynamic behaviour in the Earth's mantle can be studied.

Factors affecting plate subduction include hydrothermal factors (Yoshikawa, Okino, \& Asada, 2012), buoyancy forces, rheology of mantle minerals, stabilities of hydrous minerals, partial melting, mechanisms and rates of melt migration, kinetics of phase transformations, mechanisms of deep earthquakes, geochemical recycling, and the processes of continental collision that result from subduction (Miller, Gorbatov, \& Kennett, 2005). One fundamental problem concerns the depth to which subducted lithosphere penetrates into the mantle because this is related to the scale of mantle convection and the Earth's evolution.

\subsection{Seafloor spreading}

The seafloor spreading fabric in the Mariana Trench is obscured by the absence of magnetic anomaly lineations. Elongated topographic structures accompanied by plate 
bending exist in the oceanward slope of the Challenger Deep (Zhang, Lin, \& Zhan, 2014).

The Japanese Lineation set is a Mesozoic magnetic anomaly lineation, it exists in the East Mariana Basin, east of the Mariana Trench (Nakanishi, Tamaki, \& Kobayashi, 1992), which means that the subducting plate along the southern part of the Mariana Trench is a part of the Pacific plate formed in the Mesozoic. Other remarkable topographic feature of the Mariana Trench includes a set of many elongated ridges and escarpments accompanied by the plate bending on the outer slopes with two existing distinct strikes for these structures. The elongated structures have two strikes: $\mathrm{N} 85^{\circ} \mathrm{E}$ and the same as the trench axis; $\mathrm{N} 70^{\circ} \mathrm{E}$. Most bending-related structures except those near the trench axis, were formed by the reactivation of the seafloor spreading fabric.

The presence of cracks is another distinguished geological feature of the Mariana Trench (Ogawa, Kobayashi, Hotta, \& Fujioka, 1997). Mariana Trench has many open prominent cracks on its surface, up to a few meters deep and a few hundred meters long on the diatomaceous clayey sediment surfaces of its oceanward slopes. The shape of the cross-section is Y-shaped. More precisely, the bottom is a very narrow $(1-3 \mathrm{~km})$, yet in some places wide (several tens of kilometers) flattened.

Multiple ScatterPlot: Depths Distribution by Tectonic Plates

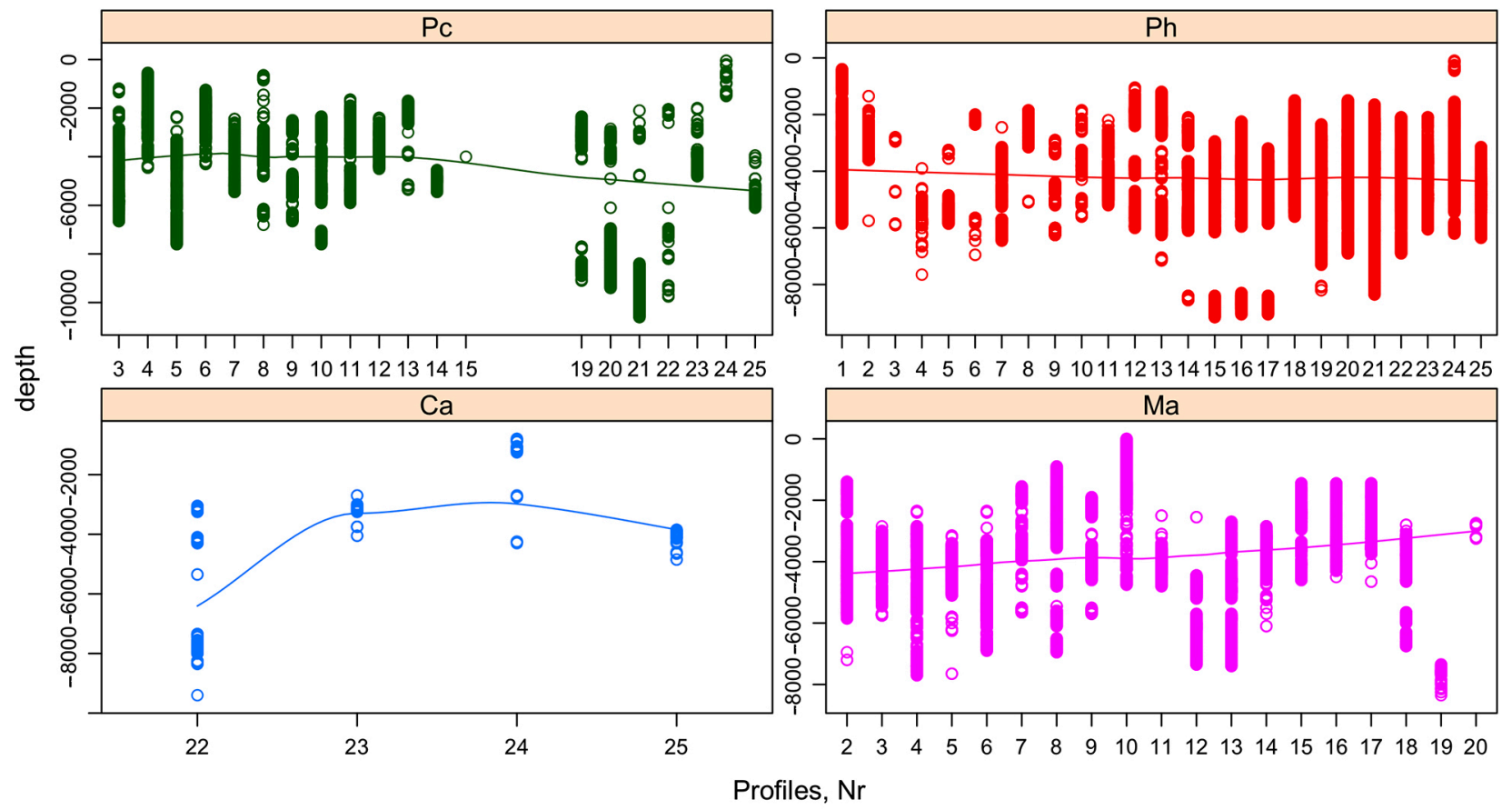

Figure 9. Multiple panel by groups: four tectonic plates, depth scope (min-max) by profiles using overlapping points
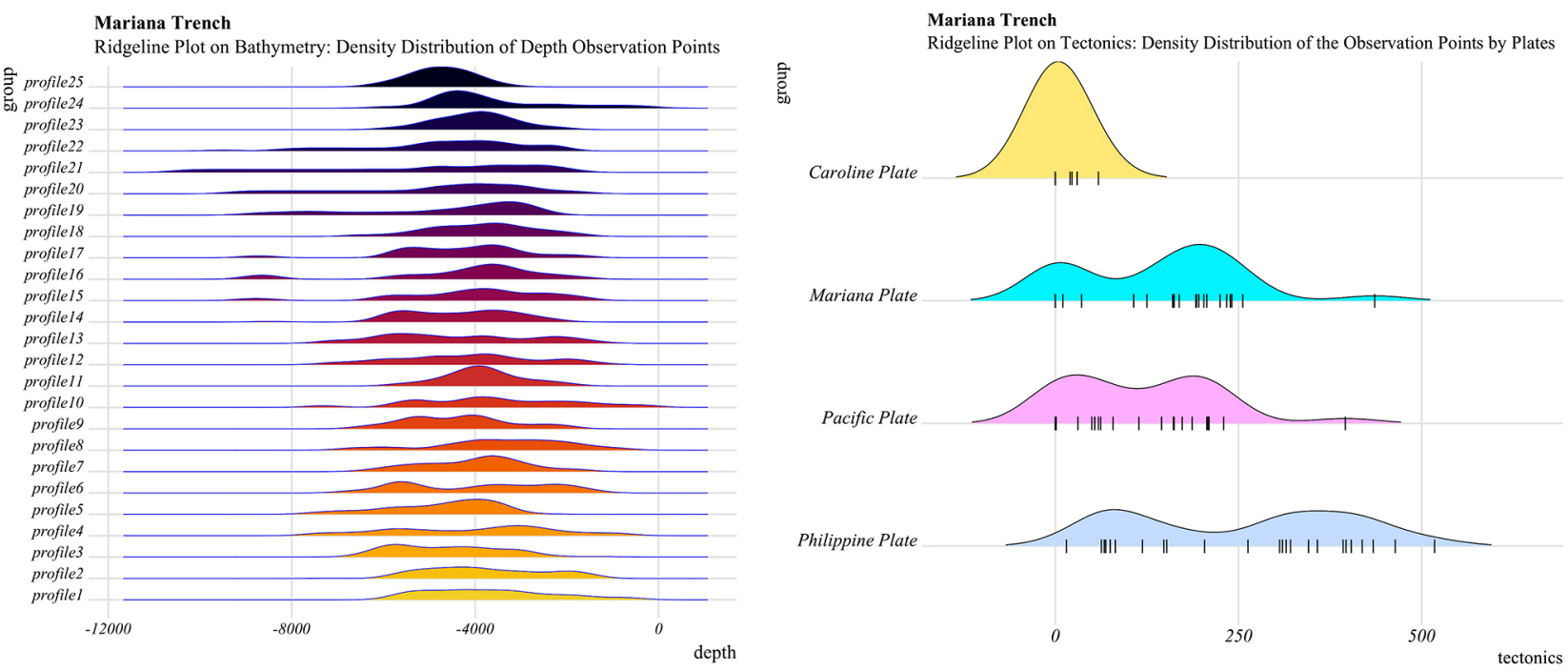

Figure 10. Ridgeline Plots by tectonic plates and bathymetric profiles 


\section{Methods and procedures}

The methodological scheme includes four general steps of the statistical analysis of the morphology of the Mariana Trench:

GIS processing of the geospatial data; creating crosssection profiles across the trench; converting coordinates to the UTM; reading depths of the observation points into the attribute table;

Statistical analysis of data distribution: calculation and visualisation for regression analysis, histograms, boxplots with outliers, theoretical and sample quantiles (QQ);

Comparative geospatial analysis by four tectonic plates: categorywise compositional bar charts on the depths distribution in observation points; multi-dimensional strip plots by four tectonic plates on maximal depth values, slope angle steepness, closeness of the igneous volcanic zones and thickness of the sediment layer;

Clustering (by k-means method), correlation ellipses, data partition, grouping and sorting.

Here the geospatial analysis aims to extract a more regular impact factors of the geologic morphology development; whereas the cluster analysis and computing correlation matrices extracts the groups and classes in a total cloud of observation points. Statistical analysis has been performed by means of $\mathrm{R}$ (Borradaile, 2003). The $\mathrm{R}$ scripting is composed of separate written codes and an algorithm for data processing.

The $\mathrm{R}$ codes presented below generate a call for various statistical formulae and algorithms used by the machine for the data processing.

\subsection{Data collection for the GIS project}

The GIS part of the research is performed in the QGIS (Figure 1). Using QGIS plugins several complex tasks were separated into smaller and more manageable components (e.g. reading coordinates, crossing profile lines, etc). Various geospatial data have been uploaded into the GIS to form the GIS project: bathymetric features of the trench, sediment thickness, location of igneous volcanic zones, distribution of the tectonic plates, etc. The GIS project has been projected into the UTM cartesian coordinate system (square $\mathrm{N}-55$ ). The GIS processing part consisted in following steps. Firstly, 25 1000-km long cross-section bathymetric profiles were digitized across the trench, as illustrated on Figure 2. The geometry of the first of the 25 profiles has been digitized across the Mariana Trench manually. Then, the line has been copied and re-entered for the next 24 profiles with a distance between every two neighbour lines of $100 \mathrm{~km}$. The numbering of the profiles goes consequently from the north to the south, from Nr. 1 to Nr. 25, respectively. Hence, every profile contained 518 measurement points, XY coordinates, elevation depth data and thematic geological data (e.g. thickness of the sediment layer) stored in an attribute table. The QGIS tool plugin "Lat Lon Tools" adding projected coordinated has been installed to activate QGIS option of adding $\mathrm{x}$ and $\mathrm{y}$ values directly to the Spreadsheet. The total dataset comprises of large amount of 12950 observations. The data were then copied into a table which was used further in this work for the statistical processing. The created profiles are visualized on Figure 3.
Mariana Trench

Ternary Diagram: Tectonic Plates

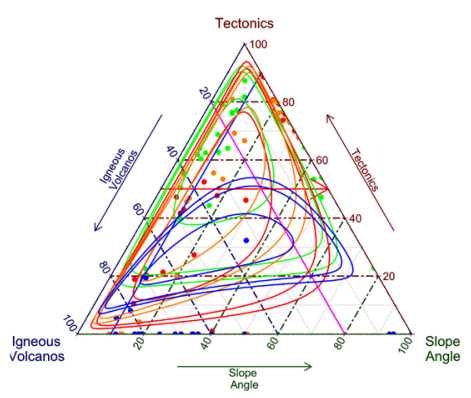

Termary Diagram: Aspect Class

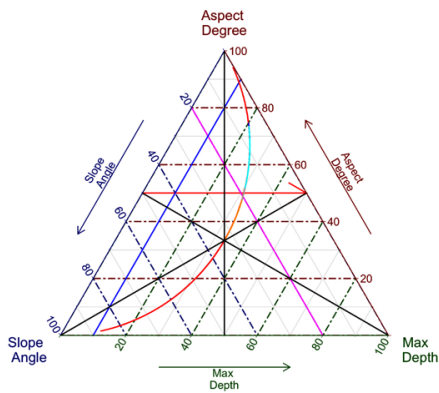

Mariana Trench

Ternary Diagram: Slope Morphology Class
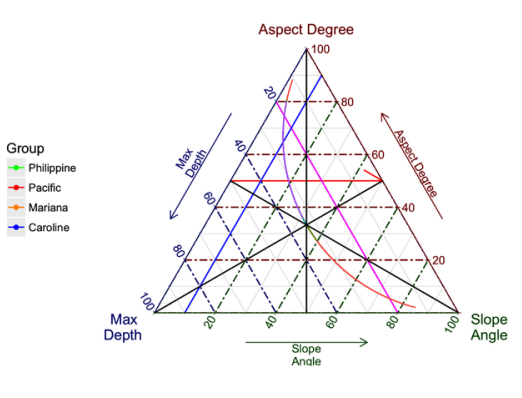

Mariana Trench. Ternary Diagram

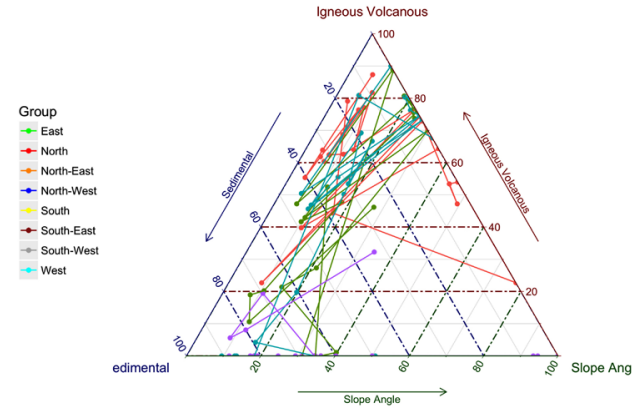

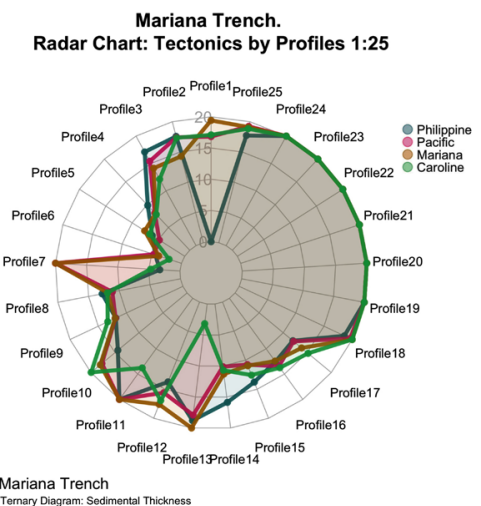

Ternary Diagram: Sedimental Thickness

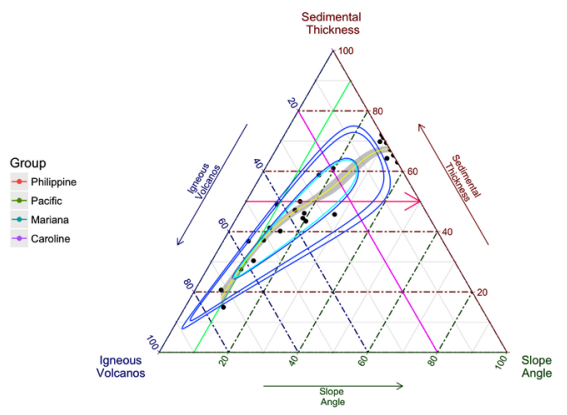

Figure 11. Ternary diagrams and radar chart: facetted plot 

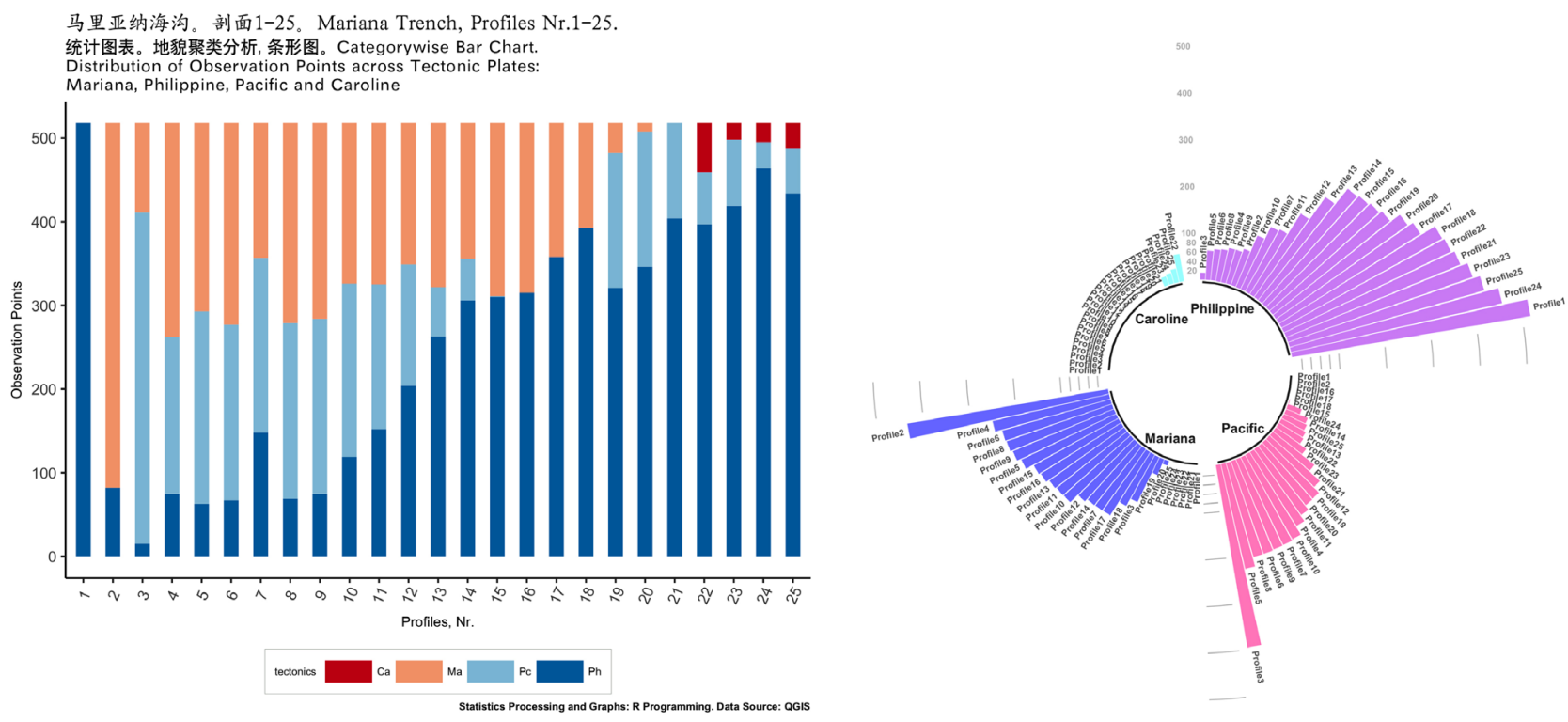

Figure 12. Category-wise distribution of depth by tectonic plates (left); circular bar plot, arranged by depth (right)

\subsection{Analysis of data distribution}

The data distribution for the bathymetric profiles has been computed using existing methods of the statistical analysis (Swan \& Sandilands, 1995) using R programming language.

\subsubsection{Histograms}

The histogram plots (Figure 4) show normal distribution of the depth observation data by the bathymetric crosssection profiles, as well as their outliers, skewness, median, mean, average, maximal, minimal and quartile distribution values. For each of 25 bathymetric profile various colours are taken for the following statistical data of depth values along the profile: "black" curves stands for normal distribution, "blue" curves - for density distribution. Vertical dashed lines: "purple" - median values, "green" mean values. The histograms have been drawn using $R$ library\{ggplot2\}. On the first step a single histogram for each of bathymetric 25 profiles was created.

The full R script to create one histogram is as follows (here: for the profile Nr. 1, further applied for every one from 25 profiles by changing the name of the file from " 01 " to " 02 ", and so on to " 25 "):

\footnotetext{
MDepths <- read.csv("Depths.csv", header=TRUE)

X01<- MDepths[,01]

$\mathrm{X} 01<-\mathrm{X} 01[$ ! is.na $(\mathrm{X} 01)]$

as.data.frame $(\mathrm{X} 01)$

dat $01<-$ data.frame $(\mathrm{X} 01)$

p01<-ggplot(dat01, aes $(\mathrm{X} 01))+$

labs(title = "Profile Nr.01", $\mathrm{x}=$ "Depths, m", $\mathrm{y}=$ "Den-

sity") +

theme(

plot.title $=$ element_text $($ family $=$ "Skia", face $=2$, size $=10)$,
}

panel.background=ggplot2::element_rect(fill = "gray91"),

legend.position $=c(.95, .95)$,legend.justification $=$ c("right", "top"), legend.box.just = "right",

legend.margin $=\operatorname{margin}(6,6,6,6)$, legend.direction $=$ "vertical", legend.background = element_blank(),

legend.key.width $=$ unit $\left(.5,{ }^{\prime} \mathrm{cm}\right.$ "), legend.key.height $=$ unit(.3,"cm"),legend.spacing = unit (.3,"cm"),

legend.box.background $=$ element_rect $($ colour $=$ "honeydew 4 ",size $=0.2$ ),

legend.text $=$ element_text(family = "Arial",

colour="black", size $=6$, face $=1$ ), legend.title $=$ element_

blank (),

strip.text.x = element_text $($ colour $=$ "white"),

panel.grid.major $=$ element_line("white", size $=0.3$ ),

panel.grid.minor $=$ element_line( $($ white", size $=0.3$,

linetype = "dotted"),

axis.text.x $=$ element_text $($ family $=$ "Arial", face $=3$,

color $=$ "gray 24 ",size $=5$, angle $=0$ ),

axis.text.y $=$ element_text $($ family $=$ "Arial", face $=3$,

color $=$ "gray24",size $=4$, angle $=90$ ),

axis.ticks.length $=\operatorname{unit}\left(.1,{ }^{\prime} \mathrm{cm} "\right)$, axis.line = element_ blank(),

axis.title.y $=$ element_text $(\operatorname{margin}=\operatorname{margin}(\mathrm{t}=20$, $\mathrm{r}=.3$ ), family = "Times New Roman", face $=1$, size $=6$ ), axis.title. $\mathrm{x}=$ element_text $($ family $=$ "Times New Ro$\operatorname{man} "$, face $=1$, size $=6, \operatorname{margin}=\operatorname{margin}(t=.2)))+$ scale_X_continuous $($ breaks $=\operatorname{pretty}($ dat $01 \$ \mathrm{X} 01$, $\mathrm{n}=4)$, minor_breaks $=\operatorname{seq}(\min ($ dat $01 \$ X 01)$, $\max ($ dat $01 \$ X 01)$, by $=500))+$ scale_y_ continuous $($ breaks $=$ scales::pretty_breaks $(\mathrm{n}=4)$, labels $=$ scales :: percent) + scale_fill_distiller(palette $=$ "RdGy") + scale_color_manual(name $=$ "Statistics:", values = c (median = "purple", mean = "green4",density = "blue", norm_dist $=$ "black")) + 
geom_histogram $($ binwidth $=200$, aes $($ fill $=$..density.., $\mathrm{X}=$ dat01\$X01,y = ..density..), color = "blue",size $=.1)+$ stat_function $($ fun $=$ dnorm, $\operatorname{args}=$ list $($ mean $=$ mean (dat01\$X01), sd = sd(dat01\$X01)), lwd =0.2, color $=$ 'black') + stat_density(

geom $=$ "line", size $=.3$, aes $($ color $=$ "density" $))+$

geom_vline $($ aes $($ color $=$ "mean", xintercept $=$ mean $(\mathrm{X} 01))$, lty $=4$, size $=.3)+$ geom_vline $($ aes $($ color $=$ "median", xintercept $=$ median $(\mathrm{X} 01))$, lty $=2$, size $=.3)+$ geom_vline $($ aes $($ color $=$ "norm_dist", xintercept $=$ dnorm $(\mathrm{X} 01))$, lty $=2$, size $=.3$ )

Using this script further 25 profiles have been created, consequently, p01, p02, .. p25.

On the next step the combination of 25 profiles on one layout (Figure 4) has been done using following R script: library(cowplot)

figure <-plot_grid(

p01 + theme(legend.position="none"),

p02 + theme(legend.position="none"),

\# continue sequently until profile Nr. 25:

p25 + theme(legend.position="none"),

labels = c(“1”, “2", “3”, “4”, “5”, “6”, “7”, “8”, “9”, “10”, “11”,

“12", “13", “14", “15”, “16”, “17”, “18”, “19”, “20”, “21”, “22”,

" 23 ", “24", “25”),

ncol $=4$, nrow $=7$ )

On the final step the annotation for the figure has been created using following $\mathrm{R}$ script:

figure_all_cowplot<- annotate_figure(figure,

top = text_grob("Mariana Trench Bathymetry: Histograms of Depth Distribution", color = "lightsteelblue4", face $=$ "bold", size $=10$ ),

bottom = text_grob("Data processing: \n R, QGIS", color $=$ "blue", hjust $=1, \mathrm{x}=1$, face $=$ "italic", size $=8$ ),

left = text_grob("Figure arranged using R, ggpubr", color $=$ "slategray4", size $=8, \operatorname{rot}=90$ ),

right $=$ text_grob ("1000- $\mathrm{km}$ length profiles", color $=$ "slategray4", size $=8$, rot $=270$ ),

fig.lab = "Profiles 1-25", fig.lab.face = "bold", fig.lab. size $=8$, fig.lab.pos $=$ "bottom.left")

ggsave (“figure_all_cowplot.pdf”, device = cairo_pdf, fallback_resolution $=300$, width $=210$, height $=297$, units $=$ "mm")

Generated plot of te 25 histograms (Figure 4) illustrates the frequency of the score occurrences in a continuous data set of the bathymetric depths that has been divided into classes showing variation in the samples of the depths, assumptions of the underlying statistical distribution of observation points values.

\subsubsection{Regression analysis}

To estimate the relationships between variables a regression analysis has been done using existing methods
(Journel, 2000). Regression analysis and facetted plot wrapping for 25 bathymetric profiles were executed by $\mathrm{R}$ library \{ggplot2\}. Using non-parametric regression analysis the conditional expectation of the dependent variable (that is depth values) given the independent variables was estimated (Figure 5). The confidence interval for each of 25 profiles has been done by four methods, visualized by the facetted plot wrapping for the series data comparison.

The regression analysis reveals how the typical value of the criterion variable changes when any one of the independent variables change, while the other independent variables are fixed. The formula describing regression equation describing the relationship between two variables used in this research is as follows:

$$
Y=a+b X+e .
$$

The literature refers (Journel, 2000) to three major applications of the regression analysis including general loess method ("glm"), loess method ("lm") and loess method ("loess"), all of which were tested in this research.

The following $\mathrm{R}$ codes were used for the four methods of the regression analysis visualized on Figure 5:

Loess_profile $11<$ - ggplot(MDF, aes $(\mathrm{x}=$ observ, $\mathrm{y}=$ profile11)) +

geom_point $($ aes $(\mathrm{x}=$ observ, $\mathrm{y}=$ profile 11 , colour $=$ "Samples", shape $=$ "Samples"), show.

legend=TRUE) +

geom_smooth (aes $(\mathrm{x}=$ observ, $\mathrm{y}=$ profile11, colour $=$ "Loess method"), method $=$ loess, se $=$ TRUE, span $=.4$, size $=.3$, linetype $=$ "solid", show. legend=TRUE) +

geom_smooth (aes $(\mathrm{x}=$ observ, $\mathrm{y}=$ profile11, colour $=$ "Glm method"), method $=$ glm, se $=$ FALSE, $\operatorname{span}=.4$, size $=.4$, linetype $=$ "dotted", show. legend=TRUE) +

geom_smooth $($ aes $(\mathrm{x}=$ observ, $\mathrm{y}=$ profile11, colour $=$ "Lm method"), method $=1 \mathrm{~m}$, se $=$ TRUE, size $=.3$, linetype $=$ "solid", show.legend $=$ TRUE $)+$ geom_quantile (aes $(\mathrm{x}=$ observ, $\mathrm{y}=$ profile11, colour = "Quantiles"), linetype = "solid", show. legend=TRUE) +

$$
\begin{aligned}
& \text { xlab("Observations") + } \\
& \text { ylab("Depths, m") + } \\
& \text { scale_color_manual(values = }
\end{aligned}
$$

c("Samples" = "seagreen", "Loess method" = "red", "Lm method" = "blue", "Glm method" = "orange", "Quantiles" $=$ "purple") $)+$ \# colours of elements

scale_shape_manual $($ values $=c($ “Samples" $=1))$ + \# shape of the point (here: Nr1 - "transparent circle")

labs(title="Mariana Trench, Profile 11.", subtitle = "Local Polynomial Regres-

sion, \nConfidence Interval, Quantiles \n(LOESS method: locally weighted scatterplot \nsmoothing for non-parametric regression)", caption = "Statistics Processing and Graphs: \nR Programming. Data Source: QGIS”) + theme() 
The quantiles were drawn by calling geom_quantile function of R. The generalized linear models (glm method) was used to give a symbolic description of the linear predictor of depth variability within each bathymetric profile, and a description of the error distribution in a range of the depth observation points. The quantiles graphically depict groups of the depth values through their distribution mode, according to the methods of the descriptive statistics. The spacings between the different parts of the box indicate the degree of the dispersion, i.e. spread, and skewness in the bathymetric data, and show outliers. The data outside the upper and lower quartiles are the outliers that are plotted as individual points. The outliers are the non-usual values of the depths indicating specific features of the geomorphology with extra deep values. The histograms show probability of the density distribution of the bathymetric observation by the profiles, i.e. how likely is that the data will belong to this or that part of the group values within certain depth range.

\subsubsection{Notched boxplots}

The notched boxplot (Figure 6) showing distribution of the depths with majority of the data from -3000 to
$-5000 \mathrm{~m}$ and outliers (the deepest points) show that from profile 1 to 16 the general depth increase with deepest values at profiles 10 and 11 . The $\mathrm{R}$ code for generating notched boxplot is as follows:

\#Part 1

\# step-1. generating dataframe from the raw table Depths.csv

MDepths <- read.csv("Depths.csv", header=TRUE, sep $=$ "',")

\# step-2. Cleaning dataframe from the от NA values MDepths_df <- na.omit(MDepths)

row.has.na <- apply(MDepths_df, 1, function(x) \{any(is. na(x))\}) \# check up the NA

sum(row.has.na) \# sup up theNA, result: [1] 0

head(MDepths_df) \# look up dataframe

\# Part 2: generating whisker boxplot using dataframe MDepths_df.

\# step-3. Adding palette, lines type, Chinese fonts.

$\mathrm{p}<-$ ggboxplot (

MDepths_df, title= "马里亚纳海沟。剖面1 1。Mariana Trench,

Profiles 1-25.",
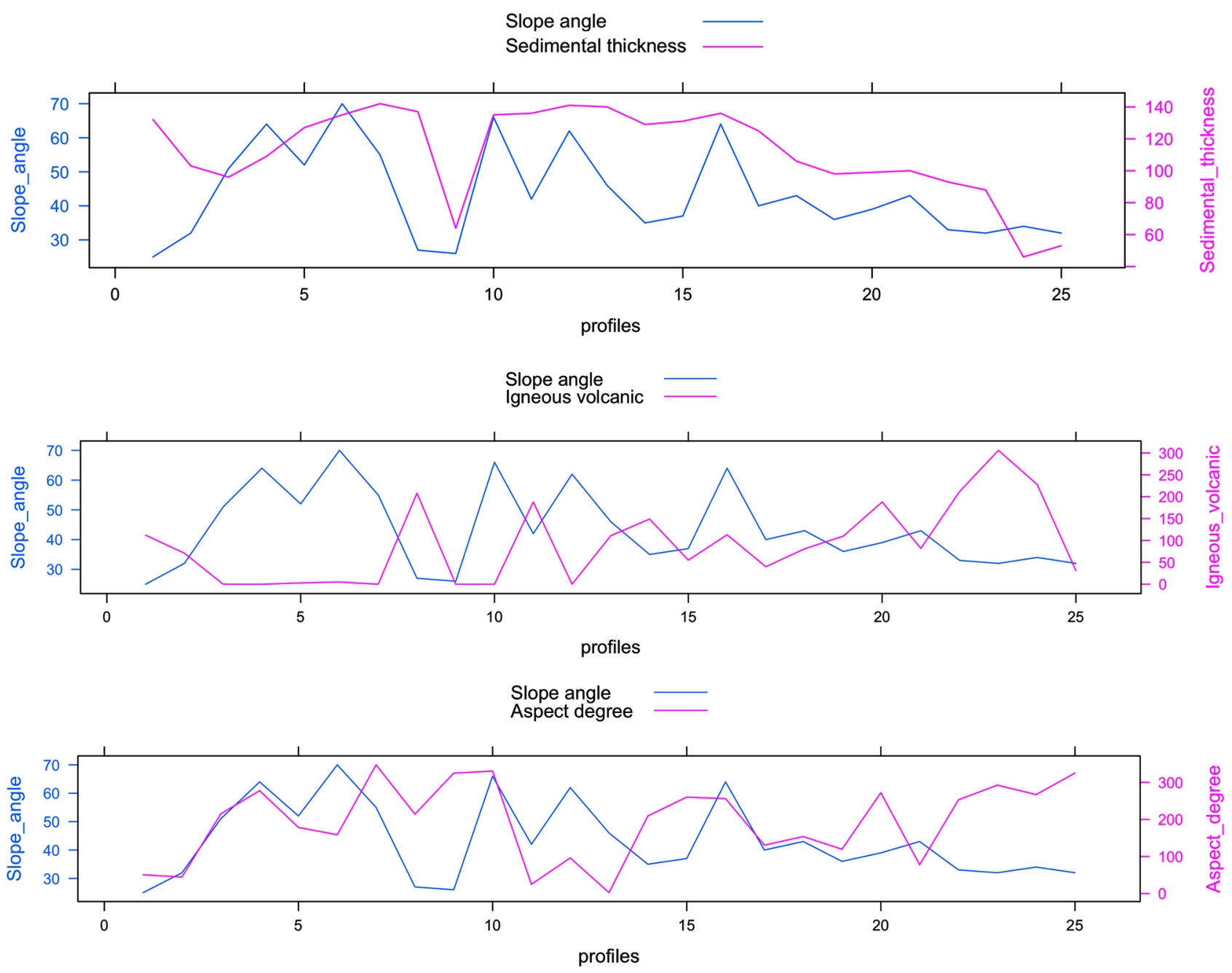

Figure 13. Double-Y-axis plot of correlation of environmental factors 
subtitle = "统计图表。箱形图 Notched Boxplot for Data Groups by 25 Profiles with Outliers)", caption = "Statistics Processing and Graphs: $\backslash n R$ Programming. Data Source: QGIS",

$$
\begin{aligned}
& \mathrm{x}=\text { "profiles", } \\
& \mathrm{y}=\text { "depths", } \\
& \text { width }=0.8, \\
& \text { notch = TRUE, } \\
& \text { fill = "profiles", }
\end{aligned}
$$

linetype $=1$, size $=.1$, outlier.colour = "grey44", palette $=c$ ("magma"), orientation = "horizontal")

\# step-4. Adding theme and palette using default format boxplot_Mariana $<-\mathrm{p}+$ theme ()

As can be seen from the notched boxplot (Figure 6), the shallowest parts of the trench are located in the profiles 24 and 25 where Mariana Trench crosses the Yap
Trench, the oceanic trench near Yap Island in the western Pacific Ocean.

\subsection{Comparative geospatial analysis by four tectonic plates}

Mariana Trench crosses four tectonic plates: Mariana, Philippine, Pacific and Caroline. Therefore, a comparative analysis of multidimensional data was performed to analyse interrelationships between the geographic location of the specific parts of the trench and factors that may impact changes in the geomorphic systems. Using existing methodology (Bretz, Hothorn, \& Westfall, 2011), various dependences between the impact factors were tested in the current research by various approaches of the comparative analysis: Figures 7, 8 and 9.

First, the distribution of various environmental factors affecting the morphology of the trench were analysed: slope angle, sedimental thickness, depth and closeness of the igneous volcanic zones to the selected bathymetric profiles (Figure 7).
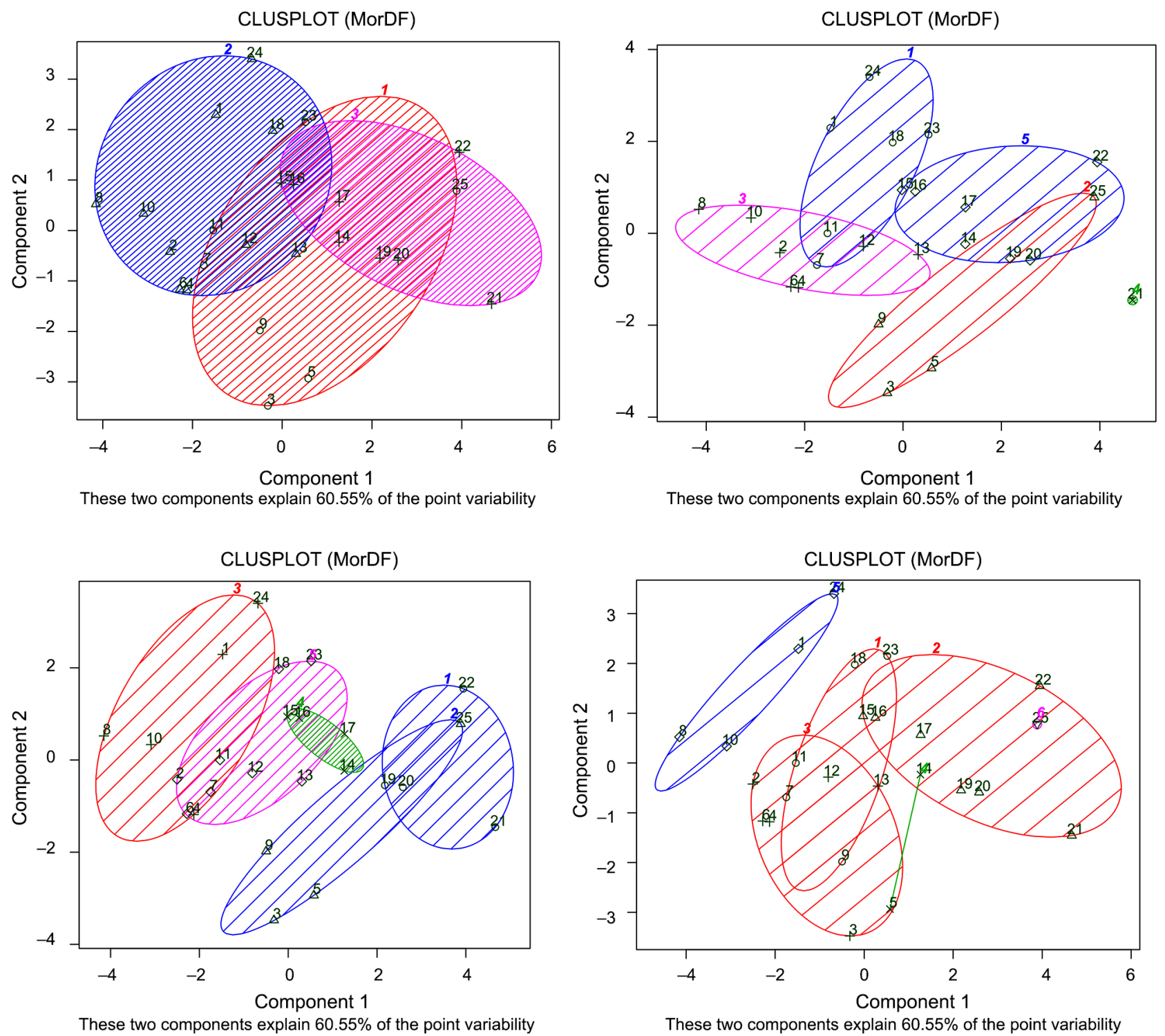

Figure 14. Results of the k-means of the clustering of the Mariana Trench profiles 

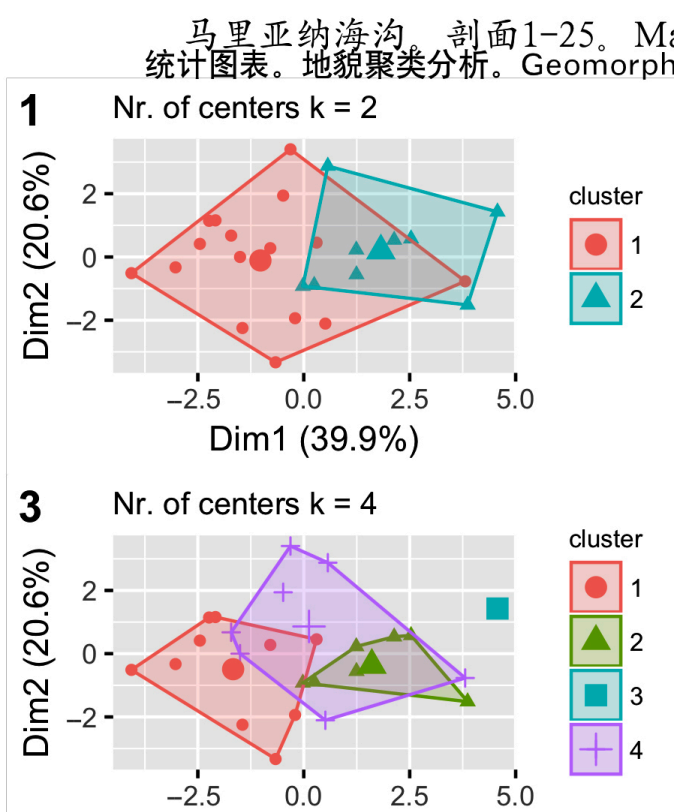

$\operatorname{Dim} 1$ (39.9\%)
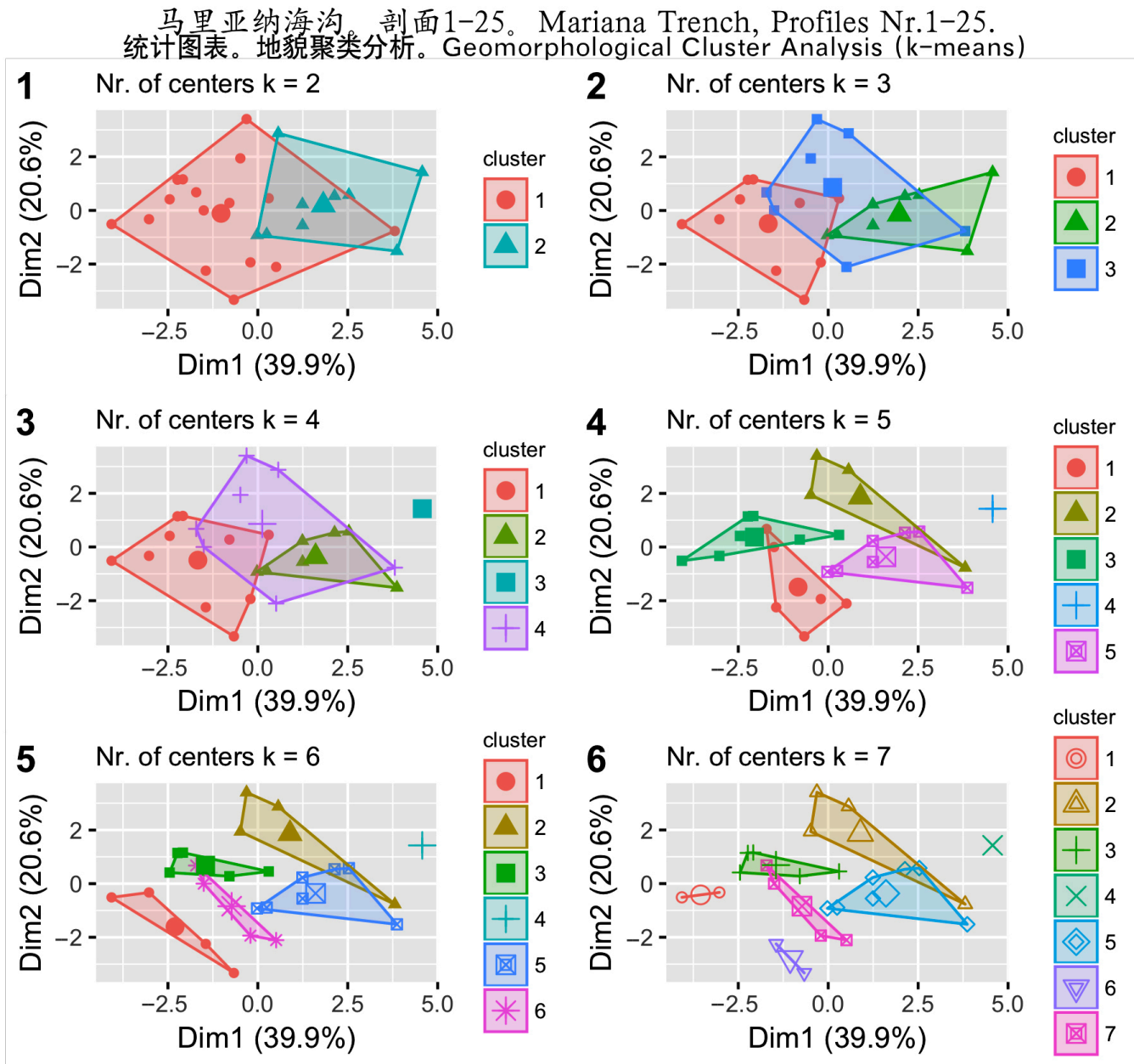

$\operatorname{Dim} 1(39.9 \%)$
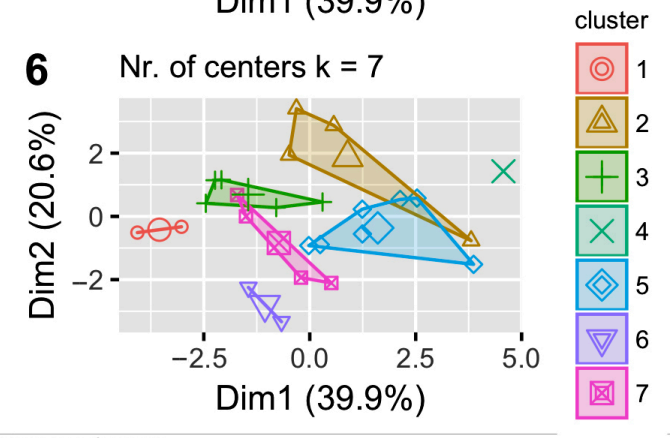

Statistics Processing and Graphs:
R Programming. Data Source: QGIS

Figure 15. Results of the k-means clustering of the Mariana Trench with different k-values

Second, the changes in the trench deepest angle, calculated as $\operatorname{tg}(\mathrm{A} / \mathrm{H})$, where $\mathrm{A}$ is the width, and $\mathrm{H}$ is the maximal depth of the profile, were calculated for each of the 25 bathymetric profiles and compared, respectively (Figure 8).

Third, the depth distribution by four tectonics plates was visualized using multiple scatter function of R (Figure 9). A pairwise correlation of the environmental factors was performed by the scripting approach of $\mathrm{R}$, which is convenient not only for looping over parts of the geomorphic model of the Mariana Trench, but also for pairwise iterating over bathymetric and geomorphic variables for the comparative analysis. Called functions "geom_smooth", "geom_point" and "geom_quantile" were used to draw smoothed conditional means and to perform regression analysis.

\subsubsection{Pairwise comparison using series of multiple strip plots divided by groups}

The multiple panel strip plots were generated in a combined plot using \{LatticeExtra2\} package of $\mathrm{R}$ by stripplot function. The distribution of the slope angle, sediment thickness, depth and closeness of the igneous volcanic zones towards Mariana Trench, by four tectonic plates is shown on Figure 7. The R code used is as follows:

$$
\begin{aligned}
& \text { \# Part-1. Create data frame } \\
& \text { \# step-1. Read in data table. Generate }
\end{aligned}
$$

data frame. Clean data frame from the NA values

MDepths <- read.csv("Morphology.csv", header=TRUE, sep $=$ "')")

MDF <- na.omit(MDepths)

row.has.na $<-\operatorname{apply}(\mathrm{MDF}, 1$, function(x)\{any(is.na(x))\}) sum(row.has.na)

head(MDF)

\# step-2. merge 4 columns with tectonic plates into one named "tectonic plates"

$\mathrm{MDFt}=\operatorname{melt}(\operatorname{setDT}(\mathrm{MDF})$, measure $=$

patterns(“^plate"), value.name $=c($ "tectonic plates"))

head(MDFt)

\# step-3. Make column with tectonic plates as

factor value (variable)

MDFt\$variable =as.factor (MDFt\$variable $)$

levels (MDFt\$variable)=c("Philippine", "Pacific", "Mari-

ana", "Caroline") \# explicitly rename 4 tectonic plates to be shown as names on $\mathrm{X}$ axis (otherwise they will be shown as numbers) 
\# Part-2. Create structured name (Title + Subtitle by various fonts) in \{LatticeExtra\} using doubleTitle function library(latticeExtra)

$$
\text { \# step-4. }
$$

doubleTitle $<$ - function $(\mathrm{a}, \mathrm{b})\{$ gTree $($ children $=$ gList ( textGrob $(a, g p=g p a r($ fontsize $=10$, fontface $=1), y=0$

$$
\mathrm{vp}=\text { viewport (layout.pos. }
$$

row=1, layout.pos.col=1)), fontface $=3), y=0$, textGrob(b, gp=gpar(fontsize $=8$,

row $=2$, layout.pos.col=1)) $\mathrm{vp}=$ viewport(layout.pos.

), $\mathrm{vp}=$ viewport (layout=grid. layout(nrow=2, ncol=1)), cl="doubletitle")

$$
\}
$$$$
\text { heightDetails.doubletitle <- function(x, }
$$
recording $=\mathrm{T})\{$

Reduce( '+', lapply(x\$children, grid:::heightDetails.text) ${ }^{\star} 2$

$$
\text { \} }
$$

\# Part-3. Strip Plot

\# step-5. Generate strip plots (vertical division of the data by four categories, here: 4 tectonic plates)

the slope angles):

$$
\text { \# variant } 5.1 \text { bathymetry (steepness of }
$$

s $1<$ - stripplot(slope_angle $\sim$ variable, data $=$ MDFt, jitter.data $=$ TRUE, pch $=20$, palette $=$ "Set $2 "$, xlab = list(label="Tectonic Plates",

$\operatorname{cex}=0.60)$,

$\operatorname{Angle}(\operatorname{tg}(\mathrm{A} / \mathrm{H}))$ ", cex=0.60),

$$
\text { ylab = list }(\text { label="Slope }
$$$$
\text { main=doubleTitle("Mariana }
$$

Trench'"'Slope Angle $(\operatorname{tg}(\mathrm{A} / \mathrm{H}))$ in 25 Profiles by Tectonic Plates"))

s1

\# variant 5.2 sedimentation (seabed sediments): s $2<$ - stripplot(sedim_thick $\sim$ variable, data $=$ MDFt, jitter.data $=$ TRUE, $\mathrm{pch}=20$,

$0.60)$,

$$
\text { xlab = list(label="Tectonic Plates", cex= }
$$

$\operatorname{cex}=0.60)$,

$$
\text { ylab = list(label="Sediment Thickness", }
$$

main=doubleTitle("Mariana

Trench'”'Sediment Thickness in 25 Profiles by Tectonic Plates"))

s2

\# variant 5.3 bathymetry (minimal depths): s3<- stripplot $($ Min $\sim$ variable, data $=$ MDFt, jitter.data $=$ TRUE, $\mathrm{pch}=20$,

$0.60)$,

$$
\mathrm{xlab}=\text { list }(\text { label="Tectonic Plates", cex }=
$$

$\operatorname{cex}=0.60)$

$$
\text { ylab = list(label="Maximal Depth", }
$$

main=doubleTitle("Mariana
Trench"',Maximal Depth in 25 Profiles by Tectonic Plates"))

s3

zones): \# variant 5.4 geology (igneous volcanic

s4<- stripplot(igneous_volc $\sim$ variable, data $=\mathrm{MDFt}$, jitter.data $=$ TRUE, $\mathrm{pch}=20$,

$$
\text { xlab = list(label="Tectonic Plates", }
$$

$\operatorname{cex}=0.60)$

$$
\text { ylab = list }(\text { label="Igneous Volcanic }
$$

Areas", cex $=0.60$ ),

$$
\text { main=doubleTitle("Mariana }
$$

Trench'”'Igneous Volcanic Zones Distribution in 25 Profiles by Tectonic Plates"))

s4

one plot \# step-6. merge all four categories on

g<- grid.arrange(s1, s2, s3, s4, ncol = 2, top $=$ grid::textGrob(label = "Statistics: $\mathrm{R}$ Programming. Data Source: QGIS”, $\quad \mathrm{x}=0.1$, hjust=0, gp=gpar(fontfamily="se rif",fontsize $=8$, fontface $=$ "bold")) )

$$
1<- \text { as_ggplot }(\mathrm{g})+
$$

draw_plot_label(label = c(“A”, “B”, “C”, “D”), size = 10, x $=c(0,0.5,0,0.5), y=c(1,1,0.5,0.5))$

\subsubsection{Multiple panels, divided by categories}

The variation of the slope angle in the deepest point and depths by 25 bathymetric profiles of the Mariana Trench according to four tectonic plates that is crosses is illustrated on Figure 8 and Figure 9. The plots have been created using $\mathrm{R}$ code in 4 steps:

initial table (.csv)

\# step-1. Creating data frame in $\mathrm{R}$ from the MDepths <- read.csv("DepthTect.csv", header=TRUE, sep $=$ "’”)

$\mathrm{MDFl}<-$ na.omit(MDepths) \# delete possible NA values row.has.na <- apply(MDFl, 1, function(x)\{any(is.na(x))\}) \# check up NA values sum(row.has.na) \# sum up NA values, receive: [1] 0 head $(\mathrm{MDFl})$ \# look up data frame.

\# step-2. Merge category groups by

classes (here: tectonics, depths, angles)

DFDT $=\operatorname{melt}(\operatorname{setDT}(\mathrm{MDFl})$, measure $=$ patterns(“^profile”, “^tectonics”, “^tg”), value.name = c(“depth", "tectonics", "trench_angle”))

head(DFDT)

\# step-3. Multiple panels by groups: $\mathrm{y} \sim \mathrm{x}$ | group generate multi-plot $\mathrm{p}<-$ xyplot (depth $\sim$ variable $\mid$ tectonics, group = tectonics, data = DFDT, type $=c($ " $p$ ", "smooth"), scales = "free", main="Multiple ScatterPlot: Depths Distribution by Tectonic Plates", xlab="Profiles, Nr")

\# step-4. Generate two variants of the multiple panels by groups: for slope angles and depths stripplot(trench_angle $\sim$ variable $\mid$ tectonics, data $=$ DFDT, layout $=c(4,1)$, jitter.data $=$ TRUE, $x l a b=$ 
“tectonic.plates", ylab = "trench_angle")

stripplot(depth $\sim$ variable $\mid$ tectonics, data = DFDT, layout $=c(4,1)$, jitter.data $=$ TRUE, xlab $=$ "tectonic.plates", ylab $=$ "depth")

The resulting graphs show variation of the slope angle in the deepest point (Figure 8) and depths (Figure 9) by 25 bathymetric profiles of the Mariana Trench by four tectonic plates: Mariana, Caroline, Pacific and Philippine.

\subsubsection{Ridgeline plots}

The ridge plots (Figure 10) were created to combine information about statistical data on 25 bathymetric profiles: arithmetic density distribution, mean and standard deviation along the profiles in a facetted way. They have lines extending horizontally from the boxes indicating variability of the Mariana Trench depths distribution across all the profiles. The code generated using \{ggridges 3 and \{ggplot2\} libraries is as follows:

\# Part-1 for 4 tectonic plates (Figure 10, right)

data frame.

\# step-1. Read-in initial table. Generate

MDF <- read.csv("Morphology.csv", header=TRUE, sep $=$ "’”)

$\mathrm{MDF}<-$ na.omit $(\mathrm{MDF})$ row.has.na <- apply(MDF, 1, function(x)\{any(is.na(x)) $\}$ ) sum(row.has.na)

head(MDF)

\# step-2. Merge columns by categories (here:

tectonics, 4 plates)

MDTt $=$ melt $($ setDT $(\mathrm{MDF})$, measure $=$ patterns(“^plate”), value.name $=c($ "tectonics”) $)$

head(MDTt)

levels (MDTt\$variable) = c("Philippine Plate", "Pacific

Plate", "Mariana Plate", "Caroline Plate")

head(MDTt)

\# step-3. Create short data frame only from 2 necessary categories: names of the 4 plates and values of the observation points:

dat $<$ - data.frame $($ group $=$ MDTt\$variable, pweight $=$ MDTt\$tectonics, tectonics $=$ MDTt\$tectonics) \# step-4. Generate ridgeline plot: $\operatorname{ggplot}($ dat, aes $(\mathrm{x}=$ tectonics, $\mathrm{y}=$ group, fill $=$ group $))+$ geom_density_ridges $($ scale $=.95$, jittered_

points=TRUE, rel_min_height $=.01$,

0.25 , point_shape $=$ “|”, point_size $=3$, size $=$

$0))+$ position $=$ position_points_jitter $($ height $=$ scale_fill_manual(values =c("lightsteelbluel", “plum1", "turquoise1", "lightgoldenrod1”)) +

马里亚纳海沟。剖面1-25。 Mariana Trench, Profiles Nr.1-25.

统计图表。地貌聚类分析。Pairwise Standard Scatter Plots of k-means Cluster Correlation

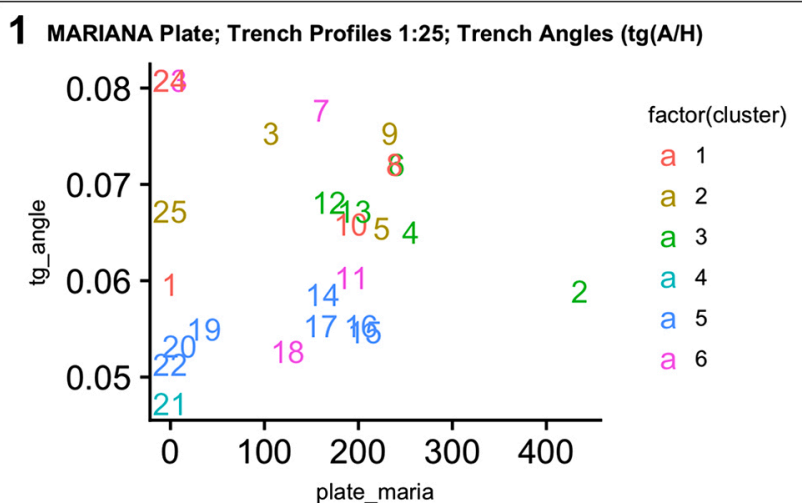

2 PHILIPPINE Plate; Trench Profiles 1:25; Trench Angles (tg(A/H)

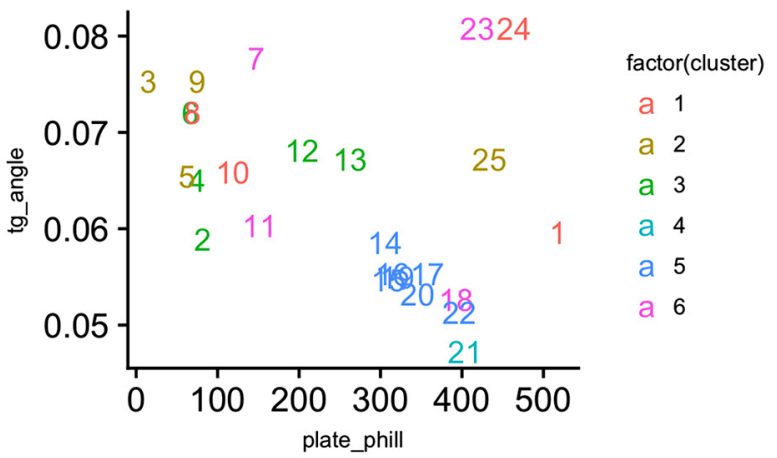

3 PACIFIC Plate; Trench Profiles 1:25; Trench Angles (tg(A/H)

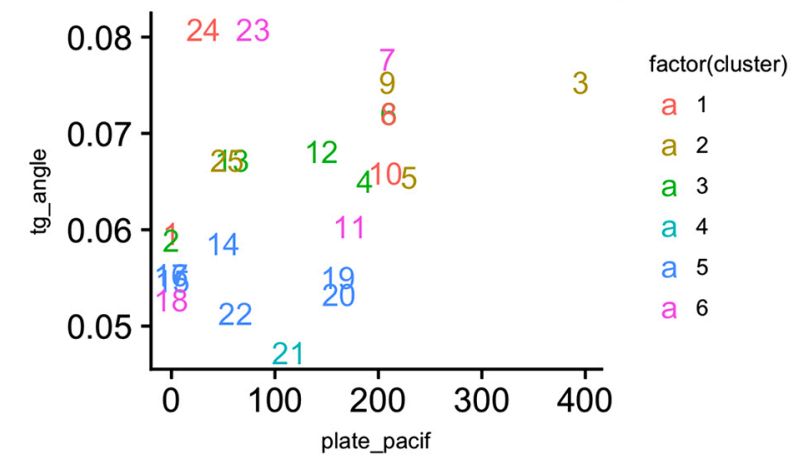

4 CAROLINE Plate; Trench Profiles 1:25; Trench Angles (tg(A/H)

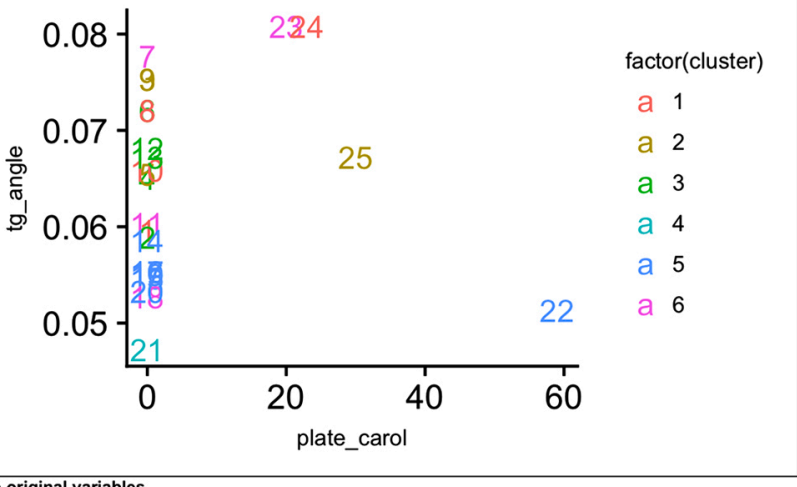

Clusters compared to the original variables
Statistics Processing and Graphs: R Programming. Data Source: QGIS

Figure 16. Classification of the k-means clustering of the Mariana Trench by tectonic plates 
theme_ridges ()$+$

theme(legend.position = "none", plot.title $=$ element_text $($ family $=$

“Times New Roman", face $=2$, size $=12$ ), plot.subtitle $=$ element_text $($ family $=$

“Times New Roman", face $=1$, size $=12$ ), axis.title.y $=$ element_text $($ family $=$

“Times New Roman", face $=1$, size $=12$ ), axis.title. $\mathrm{x}=$ element_text $($ family $=$ "Times New

Roman", face $=1$, size $=12$ ), axis.text. $x=$ element_text $($ family $=$ "Times New

Roman", face $=3$, size $=12$ ), axis.text.y $=$ element_text $($ family $=$ "Times New

Roman", face $=3$, size $=12))+$ labs (title = 'Mariana Trench',

subtitle $=$ 'Ridgeline Plot on Tectonics: Density Distribution of the Observation Points by Plates')

\# Part 2: for bathymetric depths (Figure 10, left)

dat $<$ - data.frame (group $=$ MDDl\$variable, pweight $=$ MDDl\$depth, depth $=$ MDDl\$depth)

\# step-5. Create ridgelines for bathymetry $\operatorname{ggplot}($ dat, $\operatorname{aes}(\mathrm{x}=$ depth, $\mathrm{y}=$ group, fill $=$ group $))+$ geom_density_ridges(scale $=0.95$, jittered_ points $=$ FALSE, color $=$ "blue", size $=0.2)+$ labs $($ title = 'Mariana Trench', subtitle $=$ 'Ridgeline Plot on Bathymetry: Density

Distribution of Depth Observation Points') + scale_fill_viridis(discrete $=\mathrm{T}$, option $=$ "B", direction $=-1$, begin $=.1$, end $=.9)+$ theme_ridges ()$+$ theme ()

\subsubsection{Categorywise bar plots}

To create categorywise plot (Figure 12, left) following methodology was used. First, the table was read into R:

MDepths <- read.csv("DepthTect.csv", header=TRUE, sep $=$ "',)

MDF <- na.omit(MDepths)

row.has.na <- apply(MDF, 1, function $(\mathrm{x})\{\operatorname{any}($ is.na $(\mathrm{x}))\})$ sum(row.has.na)

head(MDF)

Second, the tables columns were merged calling \{merge\} function from library(data.table) by environmental variables: depth, tectonics, slope angles. Then the new data frame (DFDT) was created:

DFDT $=\operatorname{melt}(\operatorname{setDT}(\mathrm{MDF})$, measure $=$ patterns(“^profile", “^tectonics", “^tg”), value.name = c("depths", "tectonics", "angles"))

Third, the plot function was called by the following code using created data frame in the previous step:

g <- ggplot $($ DFDT, aes $($ variable $))+$ geom_bar(aes(fill = tectonics), width $=0.5$, na.rm $=$ TRUE) + theme $($ axis.text. $x=$ element_text $($ angle $=65$, vjust $=0.6))+$ xlab("Profiles, Nr.") + ylab("Observation Points") + labs(title= "马里亚纳海勾。咅面1-25。 Mariana Trench, Profiles Nr.1-25.", subtitle = "统计图表。地貌聚类分析, 条形图。Categorywise Bar Chart.

InDistribution of Observation Points across Tectonic Plates: InMariana, Philippine, Pacific and Caroline", caption = "Statistics Processing and Graphs: R Programming. Data Source: QGIS") + scale_fill_brewer(palette = "RdBu") + theme()

To create circular barplot divided by groups the following full R code was used by :library\{tidyverse\}. Here: start from the step 3 after the fist two steps of creating the data frame to avoid repetition:

\# step-3 create short data frame from 3 values (here: value - the length of the petal of the circle) data $<$ - data.frame ( $\mathrm{id}=$ MDFt\$profile, \# profile numbers as factor value, i.e. repetition 1:25 individual = paste("Profile", seq( 1,25$),$ sep="'”), group $=$ MDFt\$variable, $\#$ here: 4 tectonic

plates

value $=$ MDFt\$tectonics) \# here: value of the sediment thickness layer (the length of the petal circle) levels $($ MDFt\$variable $)=c($ "Philippine", "Pacific", "Mariana", "Caroline") \# implicitly indicate the names of the platesto write on the axis $\mathrm{X}$

\# Order data:

data $=$ data $\%>\%$ arrange(group, value) \# step-4 create empty bar to add some space at the end of each group while visualizing the plot empty_bar=3 to_add = data.frame ( matrix (NA, empty_ bar*nlevels(data\$group), ncol(data))) colnames $($ to_add $)=$ colnames $($ data $)$

to_add $\$$ group=rep(levels(data\$group), each=empty_bar) data $=$ rbind (data, to_add)

data $=$ data $\%>\%$ arrange(group)

data $\$$ id $=\operatorname{seq}(1, \operatorname{nrow}($ data $))$ \# Part-2.

\# step-5 create labels and from the name and the y position of each label

label_data $=$ data

number_of_bar=nrow(label_data)

\# step-6 calculate the angles of the

labels

angle $=90-360 *$ (label_data\$id-0.5) /number_of_bar \# I substract 0.5 because the letter must have the angle of the center of the bars. Not extreme right(1) or extreme left (0)

label_data\$hust<-ifelse (angle $<90,1,0)$ \# distribute the labels right-left

label_data $\$$ angle $<$-ifelse $($ angle $<-90$, angle +180 , angle) \# flip angles to make them readable for visualizing purposes

\# Part-3 Draw circlal barplot \# step-7. prepare a data frame for the 
base_data $=$ data $\%>\%$

group_by(group) $\%>\%$

summarize $(\operatorname{star} t=\min (\mathrm{id})$, end $=\max (\mathrm{id})-\mathrm{emp}-$

ty_bar) $\%>\%$

rowwise() \%>\%

mutate $($ title $=$ mean $(c($ start, end $)))$

\# step-8. prepare a data frame for the grid

(scales)

grid_data $=$ base_data

grid_data $\$$ end $=$ grid_data $\$$ end $[c($ nrow(grid_data $)$,

$1:$ nrow(grid_data) -1$)]+1$

grid_data $\$$ start $=$ grid_data $\$$ start -1

grid_data $=$ grid_data[-1,]

\# step-9.

$\mathrm{p}<-$ ggplot $($ data, aes $(\mathrm{x}=$ as.factor(id $), \mathrm{y}=$ value, fill $=$ group)) +

geom_bar(aes $(\mathrm{x}=$ as.factor $(\mathrm{id}), \mathrm{y}=$ value, fill $=$

group), stat="identity", alpha $=0.5)+$

\# $\quad$ scale_fill_distiller(palette = "Set1") +

scale_fill_manual(values $=c$ ("purple", "deep-

pink", "blue", "cyan")) +

\# step-10. Add a val=100/75/50/25

lines to ensure that barplots are over it.

\# step-11: draw small additional lines

indicating scales in the empty spaces between the petals here from 25-100, further from 500 over 100.

geom_segment $($ data $=$ grid_data, aes $(\mathrm{x}=$ end, $\mathrm{y}=$ 500 , xend $=$ start, yend $=500$ ), colour $=$ "grey", alpha $=1$, size $=0.3$, inherit.aes $=$ FALSE $)+$

geom_segment $($ data $=$ grid_data, aes $(\mathrm{x}=$ end, $\mathrm{y}=$ 400 , xend $=$ start, yend $=400$ ), colour $=$ "grey", alpha $=1$, size $=0.3$, inherit.aes $=$ FALSE $)+$

geom_segment $($ data $=$ grid_data, aes $(x=$ end, $y=$ 300 , xend $=$ start, yend $=300$ ), colour $=$ "grey", alpha $=1$, size $=0.3$, inherit.aes $=$ FALSE $)+$

geom_segment (data $=$ grid_data, aes $(\mathrm{x}=$ end, $\mathrm{y}=$ 200 , xend = start, yend $=200$ ), colour $=$ "grey", alpha $=1$, size $=0.3$, inherit.aes $=$ FALSE $)+$

geom_segment(data $=$ grid_data, aes $(\mathrm{x}=$ end, $\mathrm{y}=$ 100 , xend $=$ start, yend $=100$ ), colour $=$ "grey", alpha $=1$, size $=0.3$, inherit.aes $=$ FALSE $)+$

geom_segment (data $=$ grid_data, aes $(\mathrm{x}=$ end, $\mathrm{y}$ $=80$, xend $=$ start, yend $=80$ ), colour $=$ "grey", $\mathrm{alpha}=1$, size $=0.3$, inherit.aes $=$ FALSE $)+$

geom_segment(data $=$ grid_data, aes $(\mathrm{x}=$ end, $\mathrm{y}$ $=60$, xend $=$ start, yend $=60$ ), colour $=$ "grey", alpha $=1$, size $=0.3$, inherit.aes $=$ FALSE $)+$

geom_segment(data $=$ grid_data, aes $(\mathrm{x}=$ end, $\mathrm{y}$ $=40$, xend $=$ start, yend $=40$ ), colour $=$ "grey", alpha $=1$, size $=0.3$, inherit.aes $=$ FALSE $)+$

geom_segment $($ data $=$ grid_data, aes $(\mathrm{x}=$ end, $\mathrm{y}$ $=20$, xend $=$ start, yend $=20$ ), colour $=$ "grey", alpha $=1$, size $=0.3$, inherit.aes $=$ FALSE $)+$

\# step-12. Add scale annotations showing the value of each 100/75/50/25 lines annotate("text", $x=\operatorname{rep}(\max ($ data $\$$ id $), 9), y$ $=\mathrm{c}(20,40,60,80,100,200,300,400,500)$, label $=$ c(“20", “40”, “60”, “80”, “100”, “200”, “300”, “400”, “500”), color="grey", size $=2$, angle $=0$, fontface="bold", hjust $=1$ ) $+$

ylim(-200,550) + \# ylim creates 2 diameters of the circle: outer and inner ones.

\# step-13: ylim y is 550 , because all values for the tectonic plates do not exceed 550 (i.e. outer circle)

\# here: diameter of the inner circle $=$

50

+ theme(

legend.position = "none",

axis.text $=$ element_blank () ,

axis.title $=$ element_blank () ,

plot.title $=$ element_text $($ margin $=\operatorname{margin}(t=0$,

$\mathrm{r}=0, \mathrm{~b}=0, \mathrm{l}=0)$, size $=10$, face $=$ "bold"),

plot.margin $=$ unit $(\operatorname{rep}(-1,4)$, "cm")

$+$

coord_polar()+

geom_text $($ data $=$ label_data, aes $(\mathrm{x}=\mathrm{id}, \mathrm{y}=$

value +10 , label $=$ individual, hjust=hjust), color="black", fontface $=$ "bold",alpha $=0.6$, size $=2.0$, angle $=$ label_ data $\$$ angle, inherit.aes $=$ FALSE $)+$

\# Add base line information

geom_segment $($ data $=$ base_data, aes $(\mathrm{x}=$ start, $y=-5$, xend $=$ end, yend $=-5$ ), colour $=$ "black", al-

pha $=0.8$, size $=0.6$, inherit.aes $=$ FALSE $)+$ geom_text $($ data $=$ base_data, aes $(\mathrm{x}=$ title, $\mathrm{y}=$ -18 , label $=$ group $),$ hjust $=c(1,1,0,0)$, colour $=$ "black", alpha $=0.8$, size $=3$, fontface $="$ bold", inherit.aes $=$ FALSE) $\mathrm{p}$

\subsubsection{Ternary diagrams and Radar chart}

Ternary diagrams and Radar chart were plotted (Figure 11) to show the triple correlation of several factors across tectonic plates: slope morphology classes, tectonics, igneous volcanic areas, aspect degree, slope angle, sedimental thickness. The programming $\mathrm{R}$ code for the ternary diagrams is as follows:

MT1 $<-$ ggtern $($ data $=\operatorname{MDTer}$, aes $(\mathrm{x}, \mathrm{y}, \mathrm{z}, \mathrm{color}=$ Group $))+$ theme_rgbw ()$+$ geom_point ()$+$ scale_color_manual(values $=c($ ("green", "red",

"orange", "blue")) +

labs(x="Igneous \nVolcanos,"y=”Tectonics", $z=" S$ lope \nAngle",

title="Mariana Trench",

subtitle="Ternary Diagram: Tectonic

Plates") +

geom_Tline(Tintercept $=.5$, arrow $=$ arrow () ,

colour='red') +

geom_Lline $($ Lintercept $=.2$, colour $=$ 'magenta' $)+$ geom_Rline $($ Rintercept $=.1$, colour='blue' $)+$ geom_confidence_tern() 
The radar chart (Figure 12, upper right) has been generated using following $\mathrm{R}$ code by $\{\mathrm{fmsb}\}$ library:

\#Part 1 Generating data frame

$$
\text { \# step-1. Read-in initial table with ba- }
$$

thymetic and geomorphological data

MDepths <- read.csv("Morphology.csv", header=TRUE, sep $=$ "') )

MDF <- na.omit(MDepths)

row.has.na <- apply(MDF, 1, function(x)\{any(is.na(x))\}) sum(row.has.na)

head(MDF)

classes

\# step-2. Merge group categories by

MDFt $=\operatorname{melt}($ setDT $(\mathrm{MDF})$, measure $=$

patterns(“^plate”), value.name $=c($ (tectonics”))

head(MDFt)

levels(MDFt\$variable) = c("Philippine" , "Pacific", "Mariana", "Caroline")

Plates<- c("Philippine", "Pacific", "Mariana", "Caroline") \# Part-2. Radar Chart.

library(fmsb)

set.seed(25)

\# step-3. Create mini-set of 2 values.
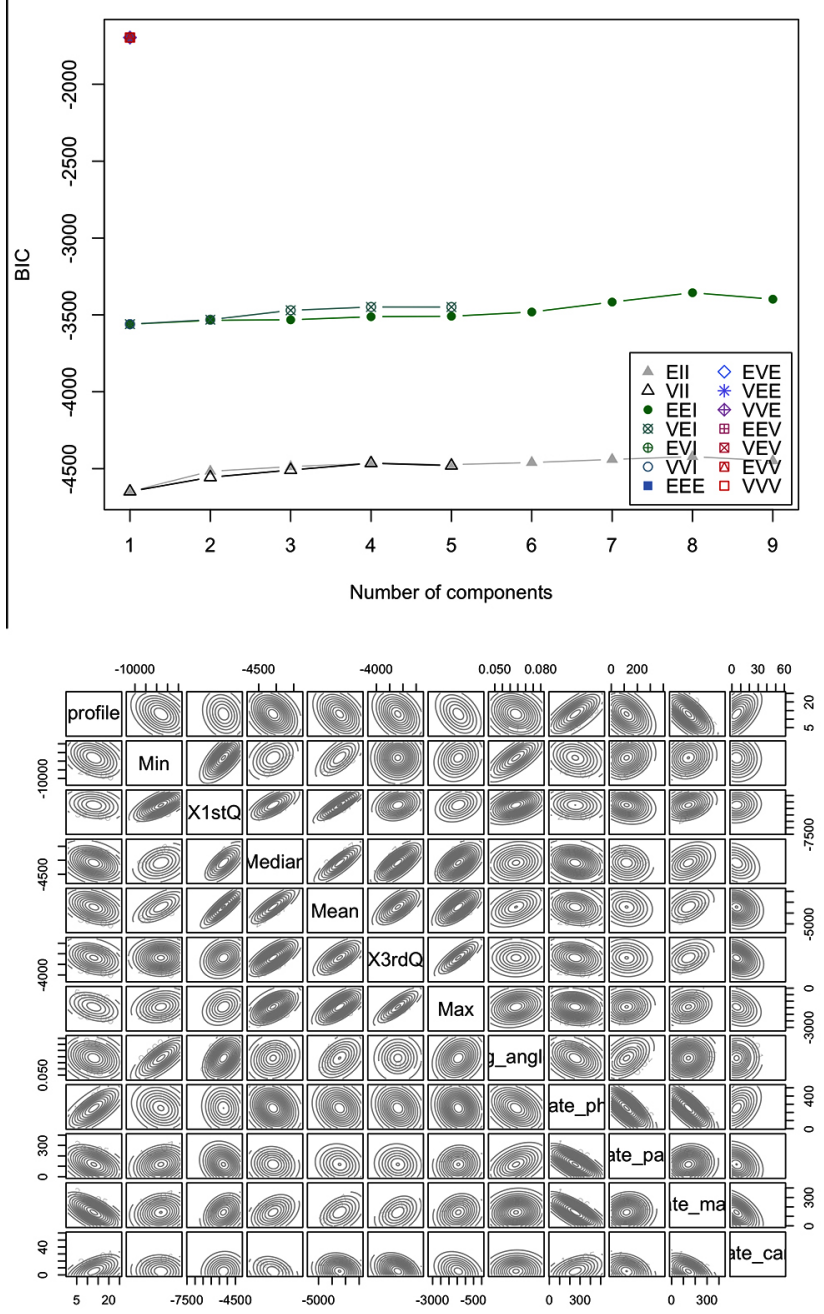

radardata $\mathrm{M}=$ as.data.frame(matrix(MDFt\$tectonics, ncol=25, nrow=4))

colnames(radardataM)<- c(paste("Profile", seq(1:25), sep="'))

rownames(radardataM)<- c("Philippine", "Pacific", "Mariana", "Caroline")

\# step-4. add 2 lines to the dataframe:

max-min of each topic

radardata $M<-\operatorname{rbind}(\operatorname{rep}(0,25), \operatorname{rep}(518,25)$, radardataM)

$$
\text { \# step-5. The default radar chart pro- }
$$

posed by the library:

radarchart(radardataM)

\# step-6. Custom the radarChart

colors_border $=\mathrm{c}(\mathrm{rgb}(0.2,0.5,0.5,0.9), \mathrm{rgb}(0.8,0.2,0.5,0.9)$, $\operatorname{rgb}(0.7,0.5,0.1,0.9), \operatorname{rgb}(0.2,0.7,0.4,0.9))$

colors_in $=c(r g b(0.2,0.5,0.5,0.1), \mathrm{rgb}(0.8,0.2,0.5,0.1)$, $\operatorname{rgb}(0.7,0.5,0.1,0.1), \operatorname{rgb}(0.2,0.7,0.4,0.1))$

radarchart ( radardataM , axistype $=1$, \#custom polygon pcol=colors_border,pfcol=colors_in , plwd $=4$, plty $=1$, cglcol="grey", cglty=1,

\begin{tabular}{|c|c|c|c|c|c|c|c|c|c|c|c|}
\hline & 0000 & & .4500 & & -4000 & & 0.0500 .08 & & $\begin{array}{l}0 \quad 300 \\
\end{array}$ & & 030 \\
\hline profile & (*) & (4) & (4) & (*) & 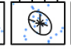 & (4) & $*$ & $\notin$ & $*$ & $\Leftrightarrow$ & * \\
\hline (7) & Min & 番 & $\%$ & (6) & $\oplus$ & (*) & 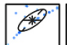 & 雨 & (7) & (*) & * \\
\hline (4) & ? & X1sto & . & 2 & $\%$ & (ब) & $\Leftrightarrow$ & ? & $\circledast$ & (4) & 3 \\
\hline 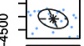 & $\Leftrightarrow$ & 馀 & hedial & 8 & ? & (6) & ? & \& & $\circledast$ & $\circledast$ & 3 \\
\hline$\Leftrightarrow$ & 0 & $?$ & 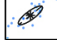 & $\|$ Mean & * & (-) & $\Leftrightarrow$ & 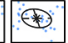 & . & $\oplus$ & (2) \\
\hline$\theta$ & 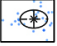 & - 6 & 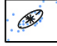 & ? & x3rdd & . & $\Leftrightarrow$ & $\Leftrightarrow$ & $\approx$ & $\theta$ & 3 \\
\hline$\Leftrightarrow$ & $\Leftrightarrow$ & 米 & 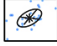 & $\approx$ & 2 & Max & $\infty$ & 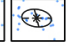 & ? & $\oplus$ & * \\
\hline 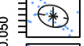 & . & (9) & $\oplus$ & 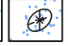 & (4) & (4) & __ang & $\|$ & $\leftrightarrow$ & (4) & e \\
\hline$\theta$ & ( ) & (4) & (4) & (1) & (4) & $\theta$ & $*$ & Ate_pr & $\approx$ & $*$ & 8 \\
\hline 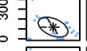 & (t) & (4) & (6) & (7) & (4) & $(4$ & $\Leftrightarrow$ & (\$) & te_pa & (*) & $\$$ \\
\hline$\theta$ & (e) & (-) & (6) & 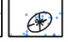 & (-) & (7) & (*) & 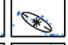 & (*) & te_me & 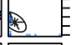 \\
\hline & -7 & |1, & & |-5000 & -30 & 0000 & 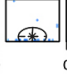 & 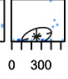 & & 番, & te_ca \\
\hline
\end{tabular}

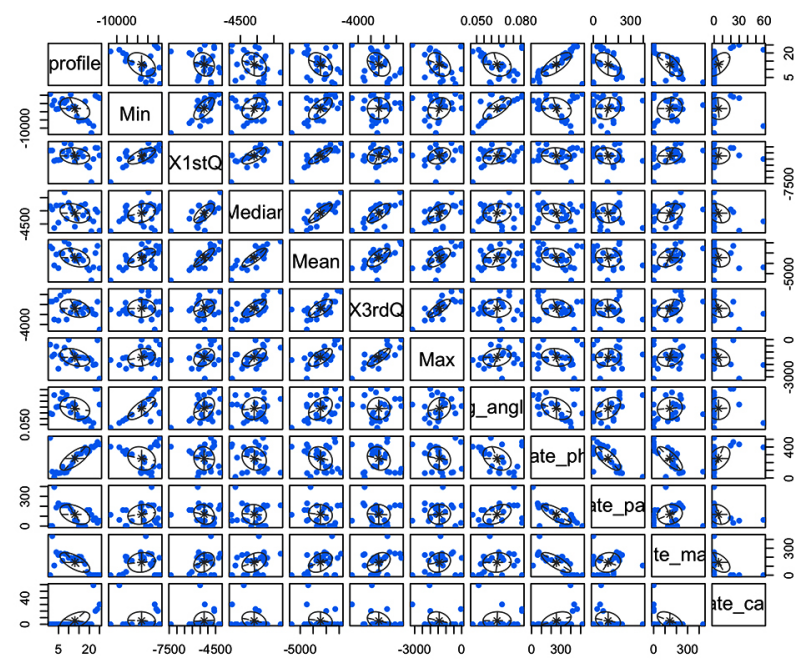

Figure 17. Correlation matrix 
axislabcol="grey", caxislabels $=\operatorname{seq}(0,20,5), \operatorname{cglwd}=0.8$, \#custom grid

vlcex $=0.8$, \#custom labels

title ="Mariana Trench. InRadar Chart: Tectonics by

Profiles 1:25", cex $=0.7$ )

legend $(\mathrm{x}=1.2, \mathrm{y}=1.0$, legend $=$ rownames $($ radardataM $[-$ $c(1,2)]$,$) ,$

$$
\text { bty }=\text { "n", pch=20, col=colors_in, text. }
$$

$\mathrm{col}=$ "grey", cex $=0.8$, pt.cex $=2$ )

\subsubsection{Pairwise double-Y-axis}

The pairwise double- $Y$ axis (Figure 13) was drawn to analyse the effect of the bi-factor correlation between the slope angle and other variables. The following script runs the model in each case, calculates the $\mathrm{X}-\mathrm{Y}$ dependences and places both graph on one plot, which his very convenient for comparative studies. The following is a code for the double-Y axis with correlations "Slope angle", "Sedimental thickness" created by library\{latticeExtra\} in $\mathrm{R}$ in 7 steps:

\# step-1. Create data set for the new data frame (only 3 necessary values: profiles, slope angle and sediments)

set.seed $(1)$

profiles $=$ MDF\$profile

Slope_angle $=$ MDF\$slope_angle

Sedimental_thickness $=$ MDF\$sedim_thick

data=data.frame(profiles, Slope_angle, Sedimental_thickness)

\# step-2 plot 'two in one' graphs: $\mathrm{p}<-$ xyplot(Slope_angle + Sedimental_thickness $\sim$ profiles, data, type $=$ "l")

objects obj $1<-$ xyplot(Slope_angle $\sim$ profiles, data, type $=$ "l" ,

$\operatorname{lwd}=1$ )

obj2 <- xyplot(Sedimental_thickness $\sim$ profiles, data,

type $=$ "l", lwd $=1$ )

\# step-6. Subscript second axis Y:

doubleYScale(obj1, obj2, add.ylab2 = TRUE)

\# step-7. Add legend:

$\mathrm{p}<-$ doubleYScale $($ obj1, obj2, text $=\mathrm{c}$ ("Slope angle", "Sedimental thickness"), add.ylab2 = TRUE)

At the end of each run, the final results are copied to the p1, p2 and p3 plot, and afterwards combined three graphs on one plot. The results of the plotting are shown on the Figure 13.

\subsection{Clustering ( $k$-means method), correlation and data grouping}

The next working step included finding correlations among the geomorphic factors that impact the morphological formation of the profiles. To this end, cluster analysis and correlation ellipses were created.

\subsubsection{Clustering (k-means method) by $R$ library\{factoextra\}}

The k-means clustering, a machine learning technique for classification performs data partition into set up number of clusters, has been done using $\mathrm{R}$ library\{factoextra\}. The goal of the k-means clustering was to perform partition of the dataset of the observations across Mariana Trench into clusters, in which each depth observation belongs to the cluster with the nearest mean, serving as a prototype of the cluster. The performed clustering is sensitive to the initial random selection of the cluster centers. Therefore, several clusters has been tested: starting from 2 to 7 . This
Mariana Trench: Correlation Ellipses
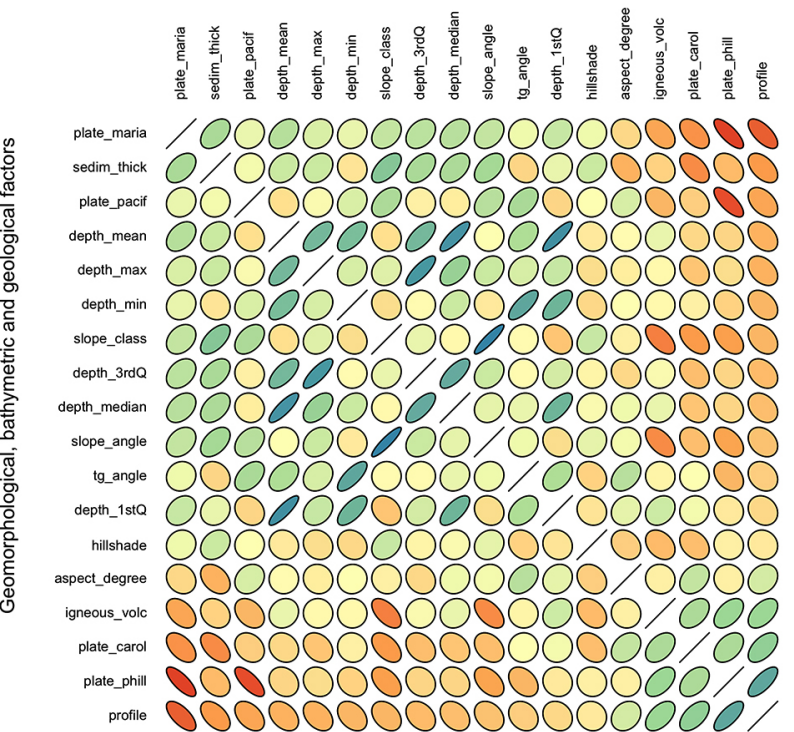

Mariana Trench: Numerical Correlation

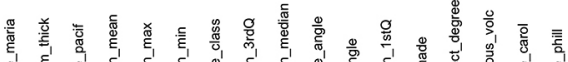

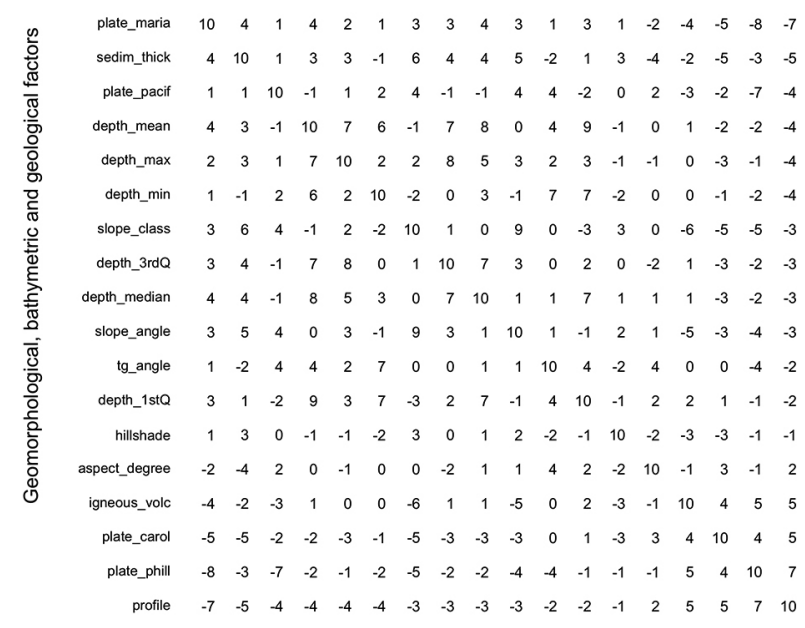

Geomorphological, bathymetric and geological factors

Figure 18. Correlation ellipses and numerical correlation of the factors affecting Mariana Trench geomorphology 
function provides a solution using a hybrid approach by combining the hierarchical clustering and the k-means methods.

The workflow procedure include following steps:

1. Computed hierarchical clustering and cutting the tree in the k-clusters using $\mathrm{R}$ code (provided below);

2. Computed pairwise standard scatter plot of k-means cluster correlation (Figure 14).

3. Computed centres ( 2 to 7 were tested, in total 6 centres) of each cluster (Figure 15);

5. Created correlation matrix (Figure 16).

6. Created groups have been tested according to their distributions by the tectonic plates (Figure 17).

The K-means cluster analysis has been performed using set of cluster centres as the initial cluster centres. The algorithm worked iteratively to assign each profile to one of the groups (2 to 7) based on the morphometric features of the Mariana Trench across these profiles that were clustered based on their geomorphic feature similarity. The results of the k-means clustering are shown on the Figure 14 and Figure 15, the correlation matrix - on the Figure 16. The pairwise standard scatter plot of $\mathrm{k}$-means cluster correlation distributed by four tectonic plates is shown on Figure 17.

The k-means cluster analysis has been done using following $\mathrm{R}$ code:
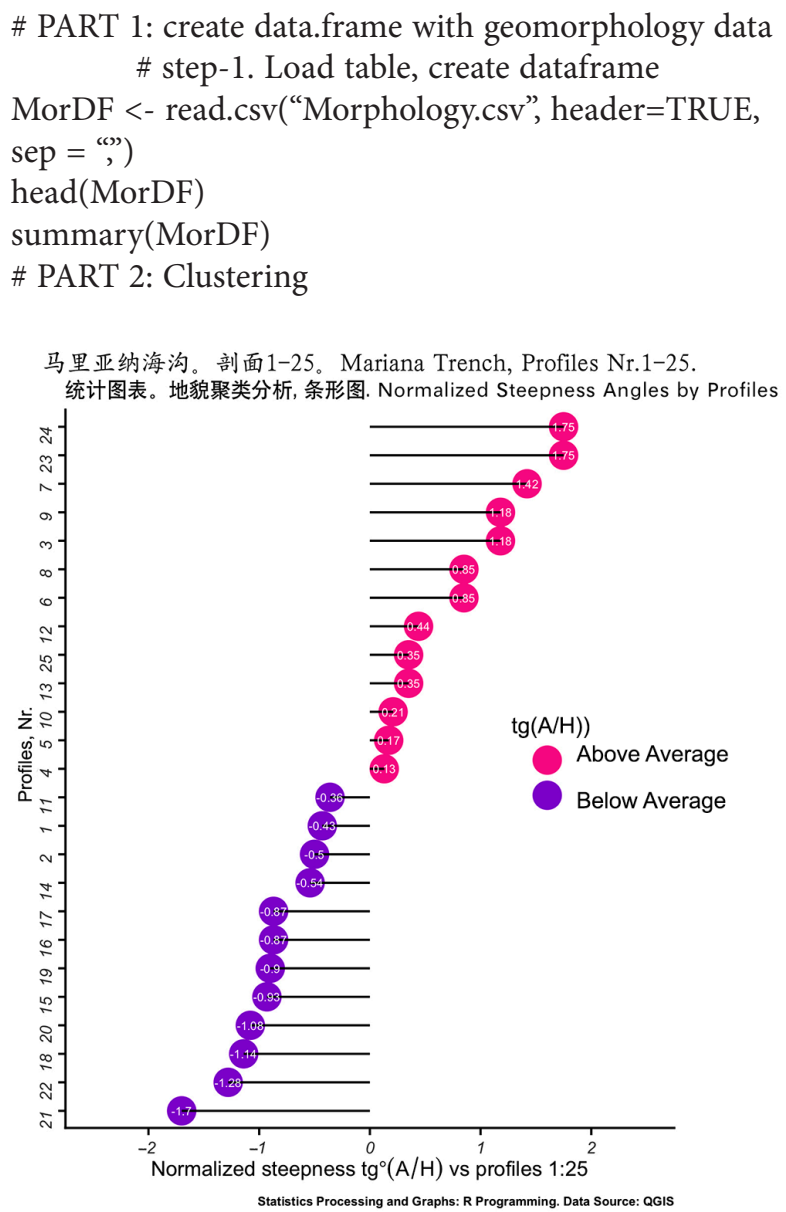

\# step-2. Create several examples of cluster analysis with various number of cluster centres (k). Here: 5 $\mathrm{k} 2$ MorDF $<-$ kmeans $($ MorDF, centers $=5$, nstart $=25$ ) $\operatorname{str}(\mathrm{k} 2 \mathrm{MorDF})$

k2MorDF

fviz_cluster(k2MorDF, data $=$ MorDF)

\# step-3. Creaing objects for each of the plots

(1to 7, here: example for plot 2):

p2 <- fviz_cluster(k2MorDF, geom = "point", data $=$ MorDF) + ggtitle( $(\mathrm{Nr}$. of centers $\mathrm{k}=2$ ") + theme(plot. title $=$ element_text $($ size $=10)$, legend.title $=$ element text $($ size $=8)$, legend.text = element_text $($ colour="black", size $=8)$ )

\# step-4. Combine all plots on one layout:

figure <-plot_grid(p2, p3, p4, p5, p6, p7, labels = c("1",

“2”, “3”, “4”, “5”, “6”), ncol = 2, nrow = 3)

\# step-5. Add legend, title and theme:

ClustersMariana6 <- figure +

labs (title = "马里亚纳海沟。剖面1-25。Mariana

Trench, Profiles Nr.1-25.,

subtitle = "统计图表。地貌聚类分析。Geomorpho-

logical Cluster Analysis (k-means)",

caption = "Statistics Processing and Graphs: $\backslash n \mathrm{n}$

Programming. Data Source: QGIS”) + theme(plot.title $=$ element_text $($ family $=$ "Kai",

face $=$ "bold", size $=12$ ),

plot.subtitle = element_text $($ family $=$ "Hei", face = "bold", size = 10)) \# 'Kai' and 'Hei' fonts indicate Chinese fonts.

The advantages of the $\mathrm{k}$-means clustering applied for Mariana Trench consists in the algorithm nature: rather than defining groups before looking at the data, clustering enabled to find and analyse the groups in the profiles that have formed organically. As the structure of the tectonics and trench geomorphic properties are rather complex, clustering facilitated data partition and grouping.

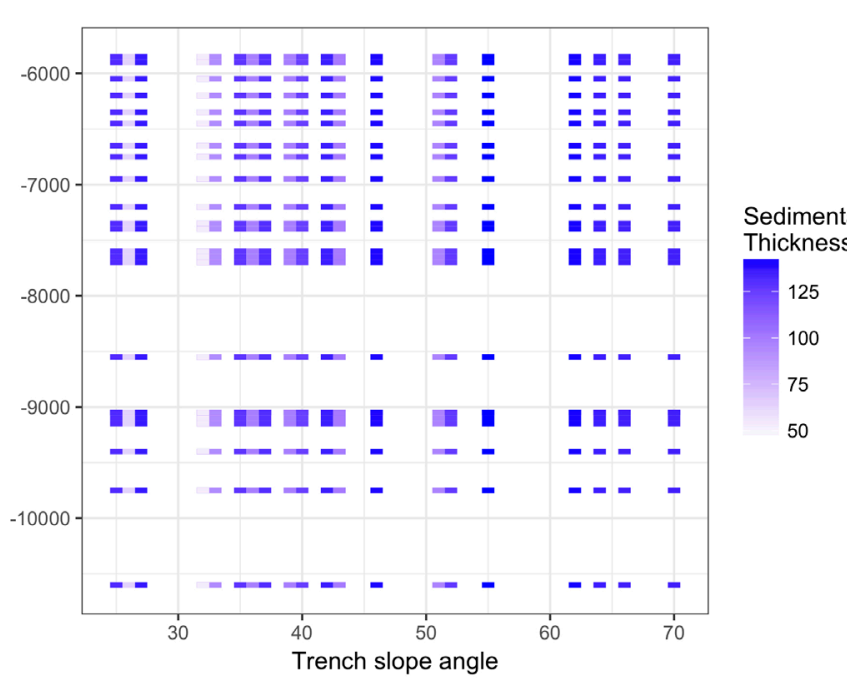

Figure 19. Normalized steepness angle (left); level plot of the sedimental thickness (right) 


\subsubsection{Correlogram ellipses and numerical correlogram}

The correlogram ellipses (Figure 18) were generated to visualize correlation between the factors affecting the morphology of the trench. The methodological approach includes calling \{ellipse $\}$ library and executing following script:

data $=\operatorname{cor}(\mathrm{MDF})$

\# step-1Build a Panel of colors by Rcolor Brew-

er

my_colors <- brewer.pal(5, "Spectral")

my_colors=colorRampPalette(my_colors) $(100)$

\# step-2. Order the correlation matrix, to dis-

tribute colours by the factors of the values

ord $<-\operatorname{order}(\operatorname{data}[1]$,

data_ord $=$ data[ord, ord $]$

\# step-3. variant-1, correlation ellipses:

plotcorr(data_ord, col=my_colors[data_ord $\left.{ }^{\star} 50+50\right]$,

$\operatorname{mar}=\mathrm{c}(1,1,1,1)$,

outline $=$ TRUE, numbers $=$ FALSE,

main = "Mariana Trench: Correlation Ellipses",

$\mathrm{xlab}=$ "Geomorphological, bathymetric and

geological factors",

ylab = "Geomorphological, bathymetric and

geological factors",

cex.lab $=0.7)$

\# step-4. variant-3, numerical correlation:

plotcorr(data_ord, col=my_colors[data_ord ${ }^{\star} 50+50$ ],

$\operatorname{mar}=\mathrm{c}(1,1,1,1)$,

outline $=$ TRUE, numbers $=$ TRUE,

main = "Mariana Trench: Numerical Correla-

tion",

xlab = "Geomorphological, bathymetric and

geological factors",

ylab = "Geomorphological, bathymetric and

geological factors",

cex.lab $=0.7)$

\subsubsection{Data partition}

Data grouping and partition has been performed by dividing angles across the trench on to classes (Figure 19, left). The level plot (Figure 19, right) shows the classes on the sedimental thickness across Mariana Trench. The data grouping has been created using libraries \{tidyverse\} and \{ggsignif\} for re-ordering and normalization of the steepness angle (Figure 20). In total 5 classes were created that include: strong slope, very strong slope, extreme slope, steep slope, very steep slope. They show classes of the steepness of the trench according to the results of the previously made cluster analysis and correlation matrix. The circular bar plot (Figure 21, left) showing percentage distribution of the sediment thickness according to the slope angle steepnesss by 25 bathymetric profiles has been drawn using $\mathrm{R}$ library\{tidyverse\}. The circulat barplot has been created using following $\mathrm{R}$ code using \{tidyverse\} library:
\# Part 1: generate data frame

MDepths <- read.csv("Morphology.csv", header=TRUE, sep $=$ "’”)

MDF <- na.omit(MDepths)

row.has.na <- apply(MDF, 1, function(x)\{any(is.na(x))\})

sum(row.has.na)

head(MDF)

\# Part 2: generate short data frame

with 3 values: profiles, sediment thickness and sequence $1: 25$

library(tidyverse)

data $<$ - data.frame ( id = MDF\$profile, \# profiles numbers as factor value, here sequence 1:25 individual $=$ paste("Profile", $\operatorname{seq}(1,25), \operatorname{sep}=$ "'”), value $=$ MDF\$sedim_thick) \# value - length of the circle petal

$$
\begin{aligned}
& \text { \# Part } 3 \text { division data into categories } \\
& \text { \# step-1 }
\end{aligned}
$$

Category <- c(paste("Profile", seq $(1,25), \operatorname{sep}=$ "') $)$

Percent $<-$ MDF\$slope_angle

\#color = add_transparency(rainbow(length(Percent)),

$0.1)$ may be used for transparency

color $=$ rainbow $($ length $($ Percent $))$

\# step-2

Category $=\operatorname{rev}($ Category $)$

Percent $=\operatorname{rev}($ Percent $)$

color $=\operatorname{rev}($ color $)$

\# Part 4 generating circle diagram us-

ing $\{$ circlize $\}$ library \# step-3

$\operatorname{par}(\operatorname{mar}=\mathrm{c}(1,1,1,1))$

circos.par("start.degree" = 90)

circos.initialize("a”, $x \lim =c(0,100))$ \# 'a' means there is one sector

circos.trackPlotRegion (ylim $=\mathrm{c}(0.5$,

length(Percent)+0.5), track.height $=0.8$,

bg.border $=$ NA, panel.fun $=$ function $(x, y)\{$

xlim = get.cell.meta.data("xlim") \# it is $c(0,100)$

for( $i$ in seq_along(Percent $)$ )

circos.lines $(x \lim , \mathrm{c}(\mathrm{i}, \mathrm{i}), \mathrm{col}=$ "\#CCCCCC”)

$\operatorname{circos} \operatorname{rect}(0, \mathrm{i}-0.45, \operatorname{Percent}[\mathrm{i}], \mathrm{i}+0.45, \operatorname{col}=$ color[i],

border $=$ "white")

circos.text(xlim[2], i, paste0(Category[i], “ - “,

Percent $[\mathrm{i}]$, “\%”), $\operatorname{adj}=c(1,0.5), c e x=.65)$

\})

circos.clear()

text $(0,0$, "Mariana Trench \nSlope Angles \nby Profiles $1: 25$ ”, col = “\#CCCCCC”, cex $=.75$ )

Created circular diagram is presented on Figure 21, left.

Finally, the logical visualization of the results as Euler-Venn diagram (Figure 21, right) is created as a visual 
illustration of the most notable geomorphological parameters which enables to analyze correlations between the environmental factors affecting trench geomorphology. The $\mathrm{R}$ code for generating Euler-Venn diagram using \{venn\} library is as follows:

\# Part 1. generating data frame:

MDepths <- read.csv("Morphology.csv", header=TRUE, sep $=$ "')")

MDF <- na.omit(MDepths)

row.has.na $<-\operatorname{apply}(\mathrm{MDF}, 1$, function $(\mathrm{x})\{\operatorname{any}(\mathrm{is.na}(\mathrm{x}))\})$ sum(row.has.na)

head(MDF)

\# Part 2. generating Euler-Venn diagram: \# case-1. Morphology and tectonic plates $\mathrm{x}<-$ list $($ Philippine $=$ MDF\$plate_phill, Pacific $=$ MDF\$plate_pacif, Mariana $=$ MDF\$plate_maria, Caroline = MDF\$plate_carol, Aspect $=$ MDF\$aspect_class, Morphology $=$ MDF\$morph_class, Slope $=$ MDF\$slope_class) = "style") venn $(\mathrm{x}$, ilabels $=$ TRUE, $\mathrm{col}=$ "navyblue", zcolor \# case-2. tectonics plates $\mathrm{xp}<-$ list $($ Philippine $=$ MDF\$plate_phill, Pacific $=$ MDF\$plate_pacif,

Mariana $=$ MDF\$plate_maria, Caroline =

MDF\$plate_carol) venn $(x p$, ilabels $=$ TRUE, ellipse $=$ TRUE, $c o l=$ "navyblue",

zcolor = "style")

\# case-3. Geomorphic parameters

$\mathrm{x} 3<-$ list $($ Depth $=$ MDF\$Min, Volcanoles =

MDF\$igneous_volc,

Sediments = MDF\$sedim_thick,

Angle $=$ MDF\$slope_angle, Hillshade = MDF\$hillshade,

Aspect $=$ MDF\$aspect_degree) $\operatorname{venn}(\mathrm{x} 3$, ilabels $=$ TRUE, col = "navyblue", zcolor $=$ "style")

\section{Results}

\subsection{Discussion of GIS and R compatibility}

The study demonstrated that the combined use of R Programming and QGIS is effective tool for the geospatial data processing and integration with further statistical analysis using R: the plugins installed for reading the coordinates, re-projecting the coordinate systems, layout visualization and attribute data conversion into xls and csv tables were efficient enough for this research. The overlapping function enables to map and visualize crossing various layers (tectonic plates, location of the volcanic igneous areas), as well as reading coordinates and depth observation points. The methodological scheme for the presented geomorphological analysis of the Mariana Trench contained a four-step approach: 1) GIS processing, 2) statistical analysis of data distribution, 3) comparative geospatial analysis, 4) data partition through cluster analysis (including k-means method and correlation ellipses).

\subsection{Findings in data distribution}

Statistical analysis revealed that the major depth observation points of the Mariana Trench are located in between the -3000 and -5000 meters (Figure 3 ), while the maximal depths reach up to -10000 in the current dataset, as can be drawn from the Figure 10: profiles 20, 21, 22 crossing mostly Philippine tectonic plate (Figure 12). Another significant change is the decrease of depth in profiles 23 , 24, 25, crossing Caroline tectonic plate, which may explain, among other factors the depth changes. Profiles Nr. 23 and 24 demonstrate the deepest depth data range.

Conversely, bathymetric profiles from 1 to 16 have gradual decrease in absolute depths (Figure 9), which can be noted in outliers sample location. A slight increase in absolute depths of the profiles \#4-8 was also observed of the Figure 8 . The majority of the observation points of

马里亚纳海沟。剖面1-25。 Mariana Trench, Profiles Nr.1-25.

统计图表。地貌聚类分析, 条形图。Geomorphological Analysis: Ranking of Profiles by Angles Steepness (Left: Unsorted; Right: Sorted and Grouped)

1

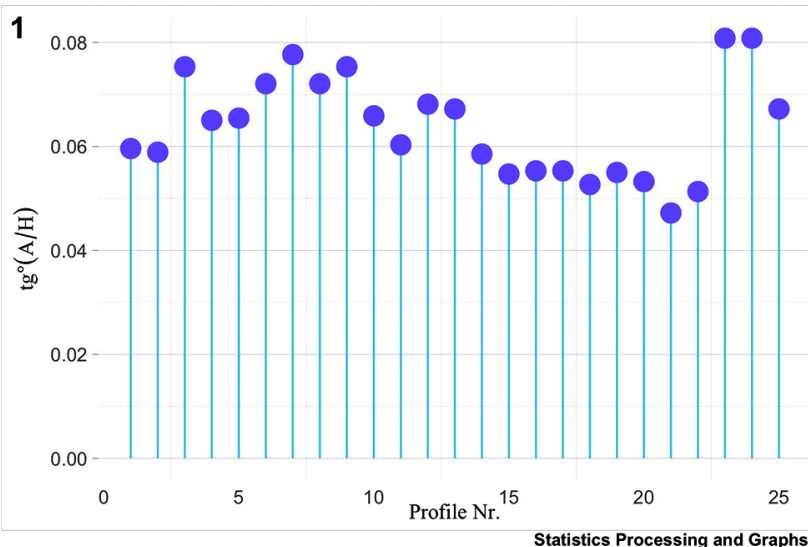

2

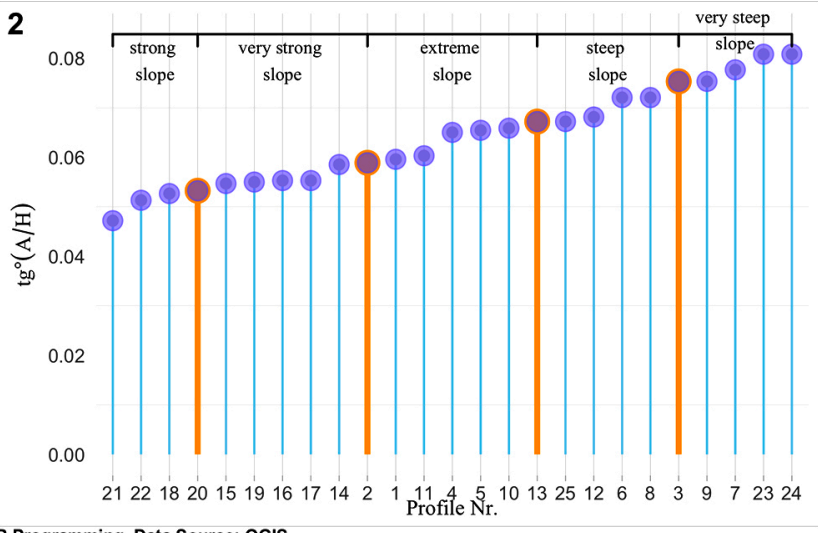

Figure 20. Trench steepness angles: unsorted (left); sorted and grouped (right) 
the mentioned above profiles crosses the Pacific tectonic plate. The changes in bathymetry may, among others be explained due to the changed environmental factors of the underlying plate. Nevertheless, sedimental thickness changes notably both within the trench by profiles (1:25) and between four tectonic plates that Mariana Trench crosses: Philippine, Pacific, Mariana and Caroline. Since the tectonic properties and attribute values of them are not identical, the comparative analysis of how the data vary across four distinctive plates was performed. As can be seen on Figure 9, right, the majority of the observation points are located in the Pacific and Philippine plate, following my Marian plate, while Caroline plate only covers a few points (red coloured, Figure 12 left).

The density distribution as shown on a combined plot (Figure 9) enables to compare the overlapping and maximal aptitude of the density curves both for the profiles and depth data distribution by four tectonic plates. Thus, the major trend of the trench angles located on the Pacific plate has downward general line trend. The Philippine tectonic plate, on the contrary, has a minimal peak by profiles \#14-21, and then moving upwards.

The highest value for Caroline. Consequently, as can be drawn from the Figure 9, Mariana plate has the highest density of depth distribution values, followed by the Philippine plate, then Pacific and Caroline, respectively. From two multiple panel by groups (Figure 7 and Figure 8) we can compare the slope angles and depth distribution by tectonic plates, respectively, one can see the unique patters of these categories by four plates.

Summaries of the variations of the local polynomial regression of the bathymetric depths of the measured samples are presented on Figure 5. Upon a close examination of these summaries it can be observed that, with a mean depth value ranging from 5000 to of 3000 on the profiles, the widths of the confidence intervals are expanding more rapidly by the profiles 12 to 15 and 19 to 22 thus indicating on the large amplitude of the depths variations in this part of the Mariana Trench. The indication is that profile depth rather being affected by the local geographic conditions caused by the location on four different tectonic plates with varying environmental conditions.

On the other hand, profiles 22 to 25 crossing Caroline tectonic plate (again refer to Figure 12, left), suggests that the absolute depth in this region change to become shallower than the profiles crossing Philippine and Pacific plates, and this phenomenon has adverse implications for deep inter-connections between these factors.

\subsection{Comparative geospatial analysis by four tectonic plates}

Studies revealed that several environmental factors have distinct effect on the Mariana Trench morphology. Among them, findings indicate (Figure 7) that sediment sources at the Mariana Trench are connected with trench tectonics where the values of the sedimental thickness lie between 120 and 140 , decreasing to $50 \mathrm{~mm}$. Thus, sedimentation processes differ significantly by four tectonic plates (Mariana, Pacific, Philippine and Caroline), due to the overall complexity of the sediment environmental processes and trigger factors and mechanisms. Among other triggers and key factors for sedimental thickness are turbidity caused by gravity sliding, biochemical deposits, closeness of seismic zones, igneous volcanic areas and differences in the sedimentation mechanisms. Besides, due to the funnelling effect, trench sediment deposits speed is faster and therefore, thickness is greater than that of the abyssal basins.
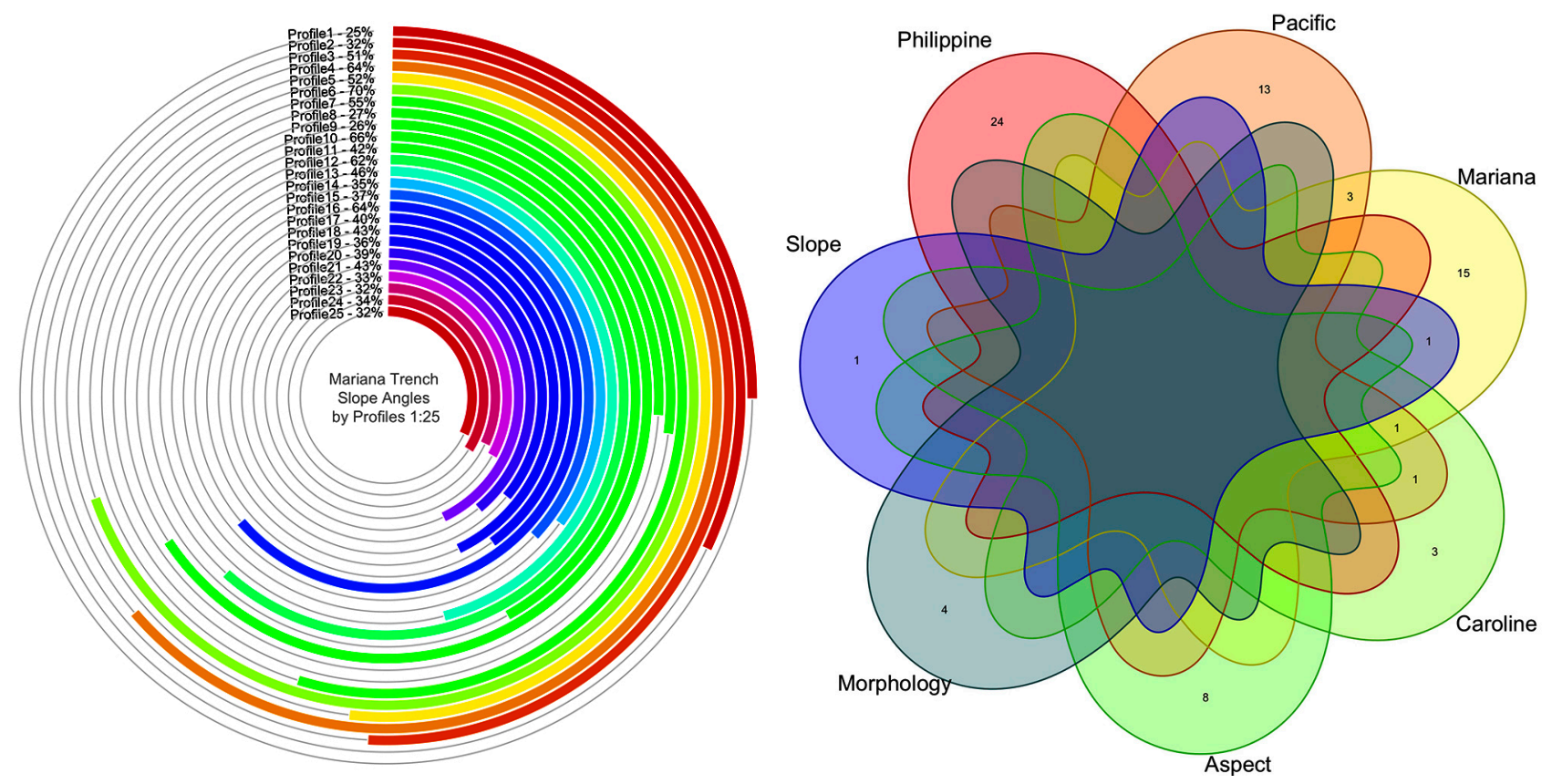

Figure 21. Circular bar plot on slope angle \% (left); logical Euler-Venn Diagram (right) 


\subsection{Findings in correlation, k-means clustering and data grouping}

The results of k-means algorithm show groups across all 25 profiles, with the number of groups represented by the variable. We tested several possible clusters from 2 to 7 , and found out the optical number, that is 5 : the cluster circle contained the optimal number of the observations and the overlapping was reasonably minimal (Figure 15 and Figure 14). The correlation matrix is presented on Figure 16 showing crossing correlations in the combination of the environmental factors. Comparison of the bi-factor in-between the factors revealed pairwise correlation (Figure 17). Pairwise comparative analysis (Figure 13) enabled to observe a marked influence on the environmental variables as bi-factors. Thus, in response to the decreasing sedimental thickness the slope angle goes in parallel; location of the volcanic igneous areas cause a cyclic repetition of the curve for the slope angles, as well as those of igneous volcanic areas have certain correlation between the slope angle and aspect degree. Therefore, according to the findings, four environmental variable factors, namely slope angle, sedimental thickness, aspect degree and location of volcanic igneous areas, are affecting the geomorphological structure of the trench.

\subsection{Findings in data partition and ranking}

Analysis of the angle steepness of the cross-section profiles along Mariana Trench revealed (Figure 19) that a bunch of bathymetric cross-section profiles form cluster groups with similar geomorphic properties divided into five groups over the study area. Thus, profiles: 21, 22, 18 and 20 have all "strong slope" $\mathrm{tg}^{\circ}$ angle degree, which is an average of 0.05 . Similarly, profiles: $15,19,16,17,14$ and 2, belonging to class "very strong slope", have an $\operatorname{tg}^{\circ}$ angle of 0.057 to 0.058 (Figure 20). When compared with third group in the study area, such as class "extreme slope" (profiles: $1,11,4,5,10$ and 13), the average slope $\operatorname{tg}^{\circ}$ angle fluctuating from 0.060 to 0.070 . The forth group is class "steep slope" (profiles: 25, 12, 6, 8 3) with a slope tg ${ }^{\circ}$ angle values from 0.070 to 0.075 . Finally, the last group is notable for the highest steepness (profiles: 9, 7, 23, 24), with average slope $\operatorname{tg}^{\circ}$ angle degree up to 0.079. Although the slope steepness is generally related to the slab subduction in this particular area, the nature of the slope expansion in the study area may also be associated with other factors such as topography, igneous closeness of the volcanic areas, and location (Pacific, Philippine, Mariana and Caroline plates).

Close examination of the slope degree statistical change revealed (Figure 20, left) that general trend has a scope of 0.03 (approximately from 0.05 to 0.08 ) of the steepness of the cross-section profiles along Mariana Trench. The reclassification of the slope angles across Mariana Trench (Figure 20, right), as well as statistical normalization have a crucial role in the analysis of the slope fluctuations. For instance, the profiles \#23 and 24 that are notable for the highest slope steepness, are located on the Philippine plate (Figure 11, right). Generally, two large classes of the normalized degree angles were observed (Figure 19): (1) below average degree (profiles \# 1,2,11, 14-19, 21-22) and (2) below average degree (all the rest).

The spatial characteristics of the steepness distribution across trench have also been shaped by the components of the igneous volcanic areas in various profiles, as understood from Figure 13: profiles \# 20, 22, 23, 24 with notable amount of igneous volcanic observation points (over 180) correlate with steepness angle. As a result of close examination, the groups of five classes of the slope steepness within trench are selected as best suitable due to the changed geomorphic properties along the trench (Figure 20, right).

Circular bar plots enabled to visualize depth distribution and slope angle values using $\mathrm{R}$ scripts (Figure 21,
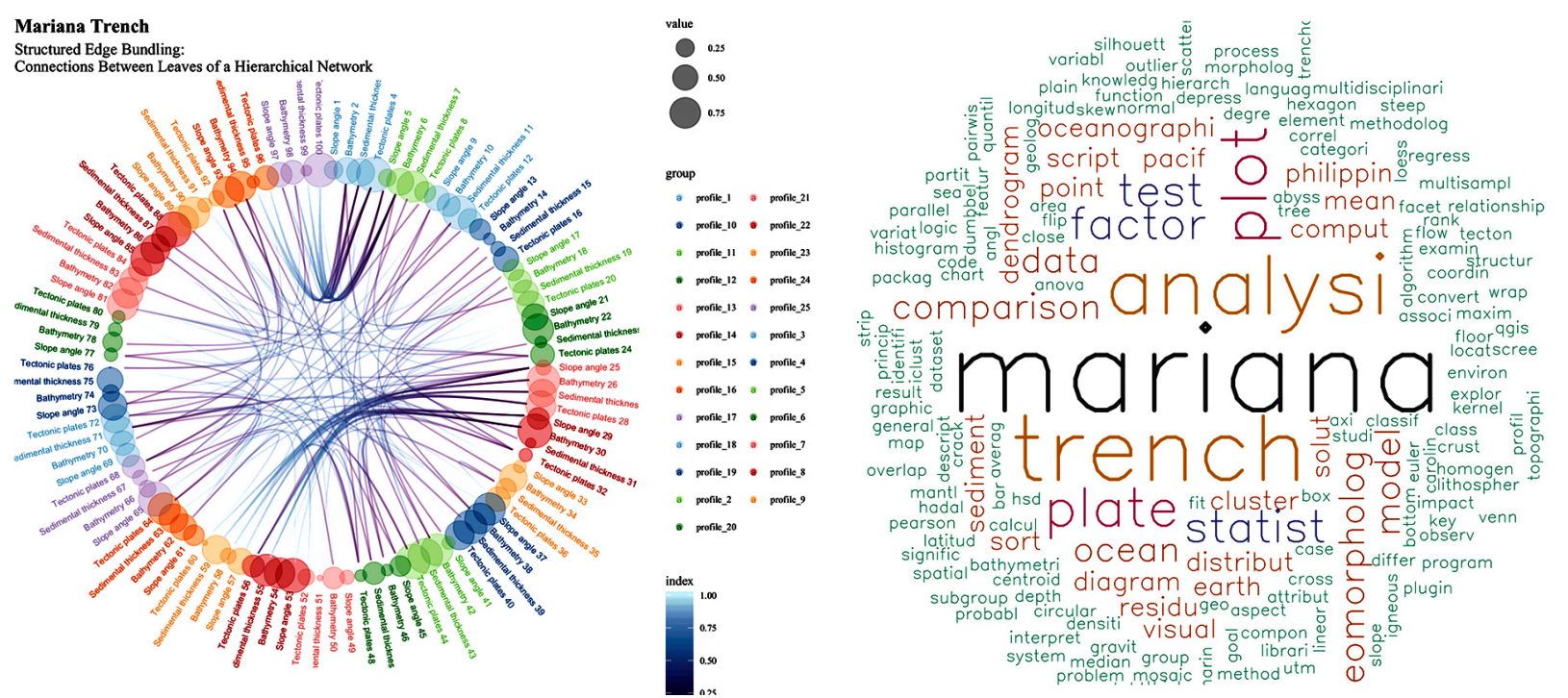

Figure 22. Logical schemes of the Mariana Trench project: inter-connections of the factors (left); word cloud (right) 
left). The Euler-Venn diagram enabled to analyse logical factors crossing and categories affecting morphology. It shows all possible logical relationships between a collection of data sets: four tectonic plates, sedimental thickness; geometric properties of the trench (that is slope angle, aspect class, hill shade group class derived as quantitative values from the GIS); morphology; volcanic closeness and depth values. As can be noticed (Figure 21, right) the area of the shape is proportional to the number of elements it contains, which is useful for explaining complex hierarchies and overlapping definitions as the case of factors affecting trench morphology. Mariana plate crosses all other plates, yet the most logical relationship has with Pacific plate. Comparing crossings between such categories as sedimental thickness and slope angle, as well as igneous zones distribution and trench slope angle. Thus, upon analysis of all possible combinations, intersections and similarities between categories, one can see the relationship between the mentioned above factors (Figure 21, right).

Finally, the integrated use of GIS, statistical methods, $\mathrm{R}$ programming applied for geological data processing aimed at a multi-disciplinary approach to study spatial distribution of the impact factors affecting trench geomorphology schematically represented on Figure 22, left. The interpretation and classification of the geospatial data by $\mathrm{R}$ were useful for estimating impact factors and triggers of the development and structure of the geomorphological structure of trench. A visual grouping of all methods, approaches and data as a cumulated flowchart is presented on Figure 22 (right) as a word cloud created by \{wordcloud\} library via R.

\section{Conclusions}

The geomorphological structure of the Mariana Trench has been impacted by various geological and geographic factors in response to the interconnected determinants of the system. Various studies have been undertaking to analyze the complexity of the system of deep ocean trenches and reveal possible impact factors (recent studies, e.g., Fernandez, \& Marques, 2018; van Rijsingen et al., 2018; Boston, Moore, Nakamura, \& Kodaira, 2017; Luo, Gieskes, Chen, Shi, \& Chen, 2017; Yoshida, 2017; Freymuth, Vils, Willbold, Taylor, \& Elliott, 2015; Ichino et al., 2015; Čížková \& Bina, 2015; Boutelier, Oncken, \& Cruden, 2014; Harris, Macmillan-Lawler, Rupp, \& Baker, 2014).

The studies of the ocean hadal trenches based on the profiles cross-section have been undertaken in the past (for instance, Murray, 1945), yet based on the technical methods of that time. Until now, little attempts has been available on application of $\mathrm{R}$ programming language and scripting libraries towards studies of ocean trenches in marine geology. Deep ocean trench formation is a very complex process that is known to be influenced by a variety of geomorphological and oceanological factors. These may not adequately explain the process of trench deep formation, its further development and what is the impact in a variety of factors affecting hadal structure of the ocean trenches. An improvement of the technical tools is therefore required in such studies.

Current analysis of the Mariana Trench geomorphology and dependence of the variability of the angle steepness from affecting factors indicate that the importance of geological factors (closeness of the nearby igneous volcanic areas, location of the part os of the trench on certain tectonic plates, angle aspect and bathymetry, depth and sediment layer thickness) are highly variable. The complex tectonic and geomorphic features of the Mariana Trench has been reviewed using a set of tested 25 cross-section profiles with unique geologic conditions located in four tectonic plates that Mariana Trench crosses. Current study demonstrated the dependences between the geomorphic factors and geology (slope angles and sedimental thickness, respectively) on the profile morphology. The quantified similarities between the distributional data distances were assessed using correlation matrices and cluster analysis ( $\mathrm{k}$-mean method).

The similarities and differences in the geomorphic structure (steepness of the slope angle degrees), geological characteristics (sediment thickness layer), geographic location of certain tectonic plates (Mariana, Caroline, Philippine and Pacific) and bathymetry (absolute minimal depths, means and median values) exist along the trench. Large gradients in variability occur within and among norther and southern part of the trench as well as in the eastern curve of its shape directed towards Magellan and Marcus-Wake transform lines. Hence, it enable to identify that geomorphological structure of the trench is mainly dominated by the following factors: the most dominated is geological factors: the closeness of the submarine volcanic areas and location of Philippine and Mariana tectonic plates, whereas sediment thickness is mainly geomorphic dominated. Thus, as a result of the undertaken study, it has been found that the middle part of the Mariana Trench (profiles: 14 up to 17), with very strong slopes, have roughly equal proportions of sediment thickness layer, which indicates that spatial locations and distributions of the volcanic areas and slope angle of the ocean trench are closely interrelated and affect sedimental thickness. The results of the research furthermore demonstrated spatial geomorphic unevenness of the Mariana Trench. Five unique regions across the trench length have been revealed, and the impact of various environmental factors affecting they morphology and characteristics were assessed by means of the applied statistics.

A contemporary perspective of $\mathrm{R}$ based geostatistical methods for the analysis of trench structure and formation is demonstrated in this research. The application of the statistical tools provided by functionality of $\mathrm{R}$ programming offer optimal prospects for better understanding of the bathymetry of the deep ocean trenches with a case study of Mariana Trench. A rigorous and quantitative quantitative data analysis performed by $\mathrm{R}$ and supported by GIS geospatial analysis methods is effective tool for 
investigation of such complex and large-scale geologic structures as deep ocean trenches. This paper demonstrated an example of such cross-disciplinary quantitative approach for geomorphological analysis. Through example of the presented research the powerful tool of the R scripting approach was shown. Using R for marine geoscience research does not only make geological modelling simpler and less error- prone. It may also facilitate more complex simulations involving, for instance, multi-dimensional modelling runs with varying geological parameters. It is also possible to apply the developed methods for extending testing of other deep ocean trenches.

Recent advances in the understanding of the hadal structure of the Mariana Trench have, so far, relied on the technologies that were unable to analyse correlations between various factors affecting its functioning and morphology. As demonstrated in this research, active using functionality of the programming languages for geoscience research provides effective tools to improve approaches to study hadal bathymetry. Development in statistical methods and using $\mathrm{R}$ scripting libraries in oceanographic studies make it possible to treat multi-source types of the marine geology data.

Full $\mathrm{R}$ codes and workflow of the statistical methods by $\mathrm{R}$ outlined in the current paper enable their repeatability and reuse for further testing in similar research. Hence, current paper contributed towards technical development of the quantitative and statistical methods in marine geomorphologic and oceanographic studies, in particular those aimed at the objective analysis of the complex interplay between the factors affecting ocean trench geomorphology.

\section{Funding}

The funding of this research has been provided by the China Scholarship Council (CSC) State Oceanic Administration (SOA), Marine Scholarship of China [Grant \# 2016SOA002, 2016] for Doctoral (PhD) studies of Polina Lemenkova.

\section{Author contributions}

All part of the research has been performed by the author (Polina Lemenkova): GIS data capture, processing and mapping, statistical analysis, programming on $\mathrm{R}$ language, plotting and visualizing graphs, writing text.

\section{Disclosure statement}

There is no competing financial, professional, or personal interests from other parties.

\section{References}

Borradaile, G. J. (2003). Statistics of Earth science data. Berlin, Heidelberg: Springer.

https://doi.org/10.1007/978-3-662-05223-5

Boston, B., Moore, G. F., Nakamura, Y., \& Kodaira, S. (2017). Forearc slope deformation above the Japan Trench megath- rust: Implications for subduction erosion. Earth and Planetary Science Letters, 462, 26-34.

https://doi.org/10.1016/j.epsl.2017.01.005

Boutelier, D., Oncken, O., \& Cruden, A. (2014). Trench-parallel shortening in the forearc caused by subduction along a seaward-concave plate boundary: In-sights from analogue modelling experiments. Tectonophysics, 611, 192-203.

https://doi.org/10.1016/j.tecto.2013.11.028

Bretz, F., Hothorn, T., \& Westfall, P. (2011). Multiple comparisons using $R$. Taylor and Francis Group, LLC.

Č́žzová, H., \& Bina, C. R. (2015). Geodynamics of trench advance: Insights from a Philippine-Sea-style geometry. Earth and Planetary Science Letters, 430, 408-415.

https://doi.org/10.1016/j.epsl.2015.07.004

Fernandez, M. O., \& Marques, A. C. (2018). Combining bathymetry, latitude, and phylogeny to understand the distribution of deep Atlantic hydroids (Cnidaria). Deep-Sea Research Part I, 133, 39-48. https://doi.org/10.1016/j.dsr.2018.01.008

Freymuth, H., Vils, F., Willbold, M., Taylor, R., \& Elliott, T. (2015). Molybdenum mobility and isotopic fractionation during subduction at the Mariana arc. Earth and Planetary Science Letters, 432, 176-186. https://doi.org/10.1016/j. epsl.2015.10.006

Gardner, J. V., Armstrong, A. A., Calder, B. R., \& Beaudoin, J. (2014). So, how deep is the Mariana Trench? Marine Geodesy, 37(1), 1-13. https://doi.org/10.1080/01490419.2013.837849

Harris, P. T., Macmillan-Lawler, M., Rupp, J., \& Baker, E. K. (2014). Geomorphology of the oceans. Marine Geology, 352, 4-24. https://doi.org/10.1016/j.margeo.2014.01.011

Ichino, M. C., Clark, M. R., Drazen, J. C., Jamieson, A., Jones, D. O. B., Martin, A. P., Rowden, A. A., Shank, T. M., Yancey, P. H., \& Ruhl, H. A. (2015). The distribution of benthic biomass in hadal trenches: A modelling approach to investigate the effect of vertical and lateral organic matter transport to the seafloor. Deep-Sea Research I, 100, 21-33. https://doi.org/10.1016/j.dsr.2015.01.010

Journel, A. G. (2000). Fundamentals of geostatistics in five lessons. Short course in geology: Volume 8. Paper presented at the 28th International Geological Congress Washington, D.C. American Geophysical Union.

Kawabe, M. (1993). Deep water properties and circulation in the western North Pacific. In T. Teramoto (Ed.), Deep Ocean circulation: physical and chemical aspects (pp. 17-37). Elsevier Science. https://doi.org/10.1016/S0422-9894(08)71315-1

Kirby, S. H., Stein, S., Okal, E. A., \& Rubie, D. C. (1996). Deep earthquakes and metastable phase transformations in subducting oceanic lithosphere. Reviews of Geophysics, 34, 261306. https://doi.org/10.1029/96RG01050

Luo, M., Gieskes, J., Chen, L., Shi, X., \& Chen, D. (2017). Provenances, distribution, and accumulation of organic matter in the southern Mariana Trench rim and slope: Implication for carbon cycle and burial in hadal trenches. Marine Geology, 386, 98-106. https://doi.org/10.1016/j.margeo.2017.02.012

Miller, M. S., Gorbatov, A., \& Kennett, B. L. N. (2005). Heterogeneity within the subducting Pacific slab beneath the IzuBonin-Mariana arc: Evidence from tomography using 3D ray tracing inversion techniques. Earth and Planetary Science Letters, 235, 331-342. https://doi.org/10.1016/j.epsl.2005.04.007

Miller, M., Kennett, B., \& Lister, G. (2004). Imaging changes in morphology, geometry, and physical properties of the subducting Pacific plate along the Izu-Bonin-Mariana arc. Earth and Planetary Science Letters, 224, 363-370. https://doi.org/10.1016/j.epsl.2004.05.018 
Murray, H. W. (1945). Profiles of the Aleutian Trench. Bulletin of the Geological Society of America, 56, 757-782. https://doi. org/10.1130/0016-7606(1945)56[757:POTAT]2.0.CO;2

Nakanishi, M., \& Hashimoto, J. (2011). A precise bathymetric map of the world's deepest seafloor, Challenger Deep in the Mariana Trench. Marine Geophysical Researches, 32(4), 455463. https://doi.org/10.1007/s11001-011-9134-0

Nakanishi, M., Tamaki, K., \& Kobayashi, K. (1992). Magnetic anomaly lineations from Late Jurassic to Early Cretaceous in the west-central Pacific Ocean. Geophysical Journal International, 109(3), 701-719.

https://doi.org/10.1111/j.1365-246X.1992.tb00126.x

Ogawa, Y., Kobayashi, K., Hotta, H., \& Fujioka, K. (1997). Tension cracks on the oceanward slopes of the northern Japan and Mariana Trenches. Marine Geology, 141, 111-123. https://doi.org/10.1016/S0025-3227(97)00059-5

Swan, A. R. H., \& Sandilands, M. (1995). Introduction to geological data analysis. Blackwell Science.

Taira, K., Kitagawa, S., Yamashiro, T., \& Yanagimoto, D. (2004). Deep and bottom currents in the Challenger Deep, Mariana Trench, measured with super-deep current meters. Journal of Oceanography, 60(6), 919-926. https://doi.org/10.1007/s10872-005-0001-y

Van Rijsingen, E., Lallemand, S., Peyret, M., Arcay, D., Heuret, A., Funiciello, F., \& Corbi, F. (2018). How subduction interface roughness influences the occurrence of large interplate earthquakes. Geochemistry, Geophysics, Geosystems, 19, 1-29. https://doi.org/10.1029/2018GC007618
Warren, B. A., \& Owens, W. B. (1988). Deep currents in the central subarctic Pacific Ocean. Journal of Physical Oceanography, 18, 529-551. https://doi.org/10.1175/1520-0485(1988)018< 0529:DCITCS $>2.0$. CO;2

Yoshida, M. (2017). Trench dynamics: Effects of dynamically migrating trench on subducting slab morphology and characteristics of subduction zones systems. Physics of the Earth and Planetary Interiors, 268, 35-53. https://doi.org/10.1016/j.pepi.2017.05.004

Yoshikawa, S., Okino, K., \& Asada, M. (2012). Geomorphological variations at hydrothermal sites in the southern Mariana Trough: Relationship between hydrothermal activity and topographic characteristics. Marine Geology, 303-306, 172-182. https://doi.org/10.1016/j.margeo.2012.02.013

Yoshioka, S., Torii, Y., \& Riedel, M. R. (2015). Impact of phase change kinetics on the Mariana slab within the framework of 2-D mantle convection. Physics of the Earth and Planetary Interiors, 240, 70-81. https://doi.org/10.1016/j.pepi.2014.12.001

Zhang, F., Lin, J., \& Zhan, W. (2014). Variations in oceanic plate bending along the Mariana trench. Earth and Planetary Science Letters, 401, 206-214. https://doi.org/10.1016/j.epsl.2014.05.032

Zhou, Z., Lin, J., \& Behn, M. D. (2015). Mechanism for normal faulting in the subducting plate at the Mariana Trench. Geophysical Research Letters, 42, 4309-4317. https://doi.org/10.1002/2015GL063917 